\title{
Modeling of combined effects of surface roughness and blowing for Reynolds-Averaged Navier-Stokes turbulence models
}

\author{
Y. Marchenay, ${ }^{1, \text { a) }}$ F. Chedevergne, ${ }^{1}$ and M. Olazabal Loumé ${ }^{2}$ \\ 1) ONERA, Multi-Physics Department for Energetics, 2 avenue Edouard Belin, FR-31055 Toulouse CEDEX 4, \\ France \\ ${ }^{2)}$ CEA-CESTA, 15 avenue des Sablières, CS60001, 33116 Le Barp CEDEX, \\ France
}

(Dated: 18 February 2021)

A new modeling strategy adapted to Reynolds-Averaged Navier-Stokes (RANS) turbulence models is proposed to predict combined effects of roughness and blowing boundary conditions. First, an analysis of experimental data is presented, leading to a specific description of the velocity profile in the logarithmic region of transpired turbulent boundary layers over rough walls. This analysis points out the deficiencies of existing roughness corrections to predict the effect of blowing in the presence of surface roughness. Indeed, these corrections tend to underestimate skin friction coefficients and Stanton numbers with addition of blowing. The failure of existing models derives from an inaccurate estimation of the velocity shift of the logarithmic law given by roughness corrections. Concretely, roughness corrections underestimate the apparent velocity shift of the logarithmic law with blowing. To recover the expected law of the wall, an additional contribution on the velocity shift, characterizing blowing/roughness interactions, is integrated to standard roughness corrections. To that end, a modification of the equivalent sand grain height, adapted to $k-\omega$ based turbulence models, is proposed to take blowing effects into account. Furthermore, an extension of Aupoix's thermal correction [B. Aupoix, International Journal of Heat and Fluid Flow 56, 160 (2015)] to blowing is presented to predict combined thermal effects of roughness and blowing. The assessment of the proposed corrections is performed using $k-\omega$ Shear Stress Transport (SST) model on a large set of experimental data and proves the relevance of the strategy for incompressible and compressible turbulent boundary layers.

Keywords: Roughness, Blowing, Turbulence, Boundary layer, Heat Transfer

\section{INTRODUCTION}

With the development of hypersonic vehicles, such as re-entry capsules or missiles, advanced technologies have been proposed to protect internal structures from the external high enthalpy flow. One of the most commonly used thermal protection systems is the ablative heat shield in which heat is dissipated by mass loss. The mass ablation rate is governed by multiple physico-chemical processes including erosion, sublimation and pyrolysis. Therefore, its description and modeling are complex and remain a subject under discussion. On the other hand, the outgassing of pyrolysis products, known as blowing, provides an additional protection against convective heat flux and, thus, reduces the total heat load undergone by the heat shield. This phenomenon is referred to as the blockage effect of blowing which creates a cooler film in the near-wall region. However, one of the major drawbacks of ablative composite materials is the development of roughness patterns (Vignoles et al. ${ }^{1}$ ) during the ablation process leading to higher heat flux and promoting the laminar-turbulent transition. As the ablation rate results from the wall heat flux, the formation of surface roughness has significant effects on the efficiency of ablative thermal protections. For this reason, the

\footnotetext{
a) Also at CEA-CESTA; Electronic mail:

Yann.Marchenay@onera.fr
}

understanding of blowing/surface roughness interactions in turbulent boundary layers is necessary to predict the aerothermal loads on such a vehicle and design the ablative heat shield accurately. The modeling of the whole ablation process and its interactions with the boundary layer is currently a challenging task and is beyond the scope of this paper. The main objective of this study is to propose a general modeling to predict combined effects of surface roughness and blowing adapted to ReynoldsAveraged Navier-Stokes (RANS) turbulence models.

Extensive studies were made on the effects of surface roughness (Ligrani and Moffat ${ }^{2}$, Flack and Schultz ${ }^{3}$ and Bons ${ }^{4}$ ). Rough wall analysis is largely based on Townsend's hypothesis ${ }^{5}$ which assumes that the outer region behavior of turbulent boundary layers is independent of wall conditions. Therefore, the propagation of the effects of roughness is confined to the inner region and preserves the logarithmic behavior of the inertial region. This assumption is considered valid if the roughness height is small compared with the boundary layer thickness (Flack et al. ${ }^{6}$ and Jiménez ${ }^{7}$ ). The first major experimental investigation dates back to the 1930s with the experiments of Nikuradse ${ }^{8}$ concerning the effects of distributed sand grain roughness on pipe flows. Nikuradse highlighted three regimes of roughness including the fully rough regime in which the effects of surface roughness become independent of the Reynolds number. In this regime, Nikuradse observed a universal surface roughness behavior driven by a single similarity parameter: the dimensionless sand grain height in wall units. 
Using this feature, Schlichting ${ }^{9}$ proposed an empirical approach to model the effects of surface roughness on skin friction. This approach, known as the equivalent sand grain approach, is the most common method for industrial applications in view of its simplicity. First, the real surface roughness is related to an equivalent sand grain roughness which yields the same increase of skin friction in Nikuradse's fully rough regime. Several authors (Dirling ${ }^{10}$, Sigal and Danberg ${ }^{11}$ and van Rij et al. ${ }^{12}$ ) proposed correlations based on geometrical parameters such as roughness height, shape and density to estimate the equivalent sand grain height. Then, RANS turbulence models are modified to mimic the roughness effect by enhancing the turbulent eddy viscosity in the wall region (Wilcox ${ }^{13}$, Hellsten and Laine ${ }^{14}$, Knopp et al. ${ }^{15}$ and Aupoix $\left.{ }^{16}\right)$. As the Reynolds analogy is no longer valid using the equivalent sand grain approach, attempts to predict heat transfer by assuming a constant turbulent Prandtl number were mostly unsuccessful. The reason comes from the nature of the drag increase caused by surface roughness. In the fully rough regime, the roughness drag is mainly characterised by a pressure drag on the roughness elements. In contrast, the equivalent sand grain approach reproduces the drag increase by enhancing the turbulence in the wall region. Consequently, heat transfers on rough wall are overpredicted in most cases using the Reynolds analogy (i.e. a constant turbulent Prandtl number) with the equivalent sand grain approach. To solve this problem, Aupoix ${ }^{17}$ proposed a modification of the turbulent Prandtl number involving another geometrical parameter: the corrected wetted surface.

Similarly to surface roughness, wall transpiration (blowing or suction) was extensively studied in the context of turbulent boundary layers. The interest in wall transpiration is motivated by potential turbulent flow control $^{18}$ (drag reduction, laminar-turbulent transition delay, boundary layer separation) and thermal protection applications ${ }^{19}$ (transpiration cooling). By deriving a mathematical relationship (FIK identity), Fukagata et $a l .{ }^{20}$ pointed out the different effects of uniform blowing on skin friction. The dominant contribution of blowing comes from the mean wall normal convection leading to a strong drag reduction. Conversely, a minor contribution of the drag increasing is reported and derives from the enhancement of turbulent motions in the near wall region. Furthermore, an additional effect of wall blowing is the alteration of the velocity profile in the inertial region. Stevenson ${ }^{21}$ is the first author to propose a general description of the law of the wall in the presence of suction or blowing. Stevenson's law is characterized by a modification of the slope of the log-law (bilogarithmic form) in which the integration constant $C$ is equal to its non-blowing value. Thereafter, many authors (Danberg $^{22}$, Squire ${ }^{23}$ and Voisinet ${ }^{24}$ ) showed that the integration constant should be considered as a function of the blowing velocity without questioning the bilogarithmic form. Recently, Vigdorovich ${ }^{25}$ proposed a generalization of Stevenson's law of the wall by considering the asymptotic behavior of the velocity profile. Relatively few numerical works derived strategies to account for blowing in RANS turbulence models. Most of the RANS corrections are ad hoc modifications based on the increase of the turbulent eddy viscosity in the wall region. The problem lies in the fact that the majority of RANS turbulence models tend to underpredict the enhancement of near wall turbulent motions in the presence of blowing. Consequently, the predicted skin friction is generaly lower than experimental values for medium/high blowing ratios. According to Andersen's data ${ }^{26}$, Wilcox ${ }^{13}$ proposed a modification of the wall specific dissipation to adapt $k-\omega$ based models to blowing conditions. Belletre et al. ${ }^{27} \mathrm{im}-$ proved the behavior of turbulence models by mimicking the porous wall conditions. Hink et al. ${ }^{28}$ derived an extension of Spalart-Allmaras turbulence model ${ }^{29}$ to high blowing ratios by exploiting the similarity of Wilcox's corrections for surface roughness and blowing. More recently, Chedevergne and Marchenay ${ }^{30}$ proposed a general strategy to recover Stevenson's law of the wall, improving the predictions of RANS turbulence models.

Despite a significant number of studies on surface roughness and blowing effects, only few works investigated the combined effects of roughness and blowing on turbulent boundary layers (Healzer et al. ${ }^{31}$, Pimenta et al. $^{32}$, Coleman et al. ${ }^{33}$, Schetz and Nerney ${ }^{34}$, Voisinet ${ }^{24}$, Miller et $a .^{35}$ and Holden ${ }^{36}$ ). In most experiments, the effect of blowing for smooth and rough walls exhibits a similar behavior. The presence of blowing decreases skin friction coefficients and Stanton numbers with the increase of the blowing rate no matter what the level of surface roughness. Pimenta ${ }^{32}$ pointed out some "surface roughness characterictics" in the wall region of smooth transpired boundary layers. Both surface roughness and blowing tend to decrease the viscous sublayer thickness and the turbulence anisotropy near the wall. Stevenson's law of the wall is generally observed but shifted downwards due to the effect of surface roughness. The recent Direct Numerical Simulation (DNS) of Mori et al. ${ }^{37}$ suggests that Stevenson's law of the wall remains valid in the presence of surface roughness by adding a roughness shift term. Furthermore, Voisinet ${ }^{24}$ indicated that the prediction of the combined effects of roughness and blowing is more complex than the superposition of each independent theory. Modeling attempts were proposed by some authors (Healzer et al..$^{31}$, Christoph and Pletcher ${ }^{38}$ and Ilegbusi ${ }^{39}$ ) with varying degrees of success. To our knowledge, all existing corrections remain restricted to the mixing length approach or to the wall function strategy and, thus, are not suited to standard RANS turbulence models. For this reason, the present paper is dedicated to the derivation of new roughness/blowing corrections adapted to RANS turbulence models.

The paper is organized as follows. Some key characteristics of transpired turbulent boundary layers over rough walls are presented in section II. Existing roughness and blowing corrections are also reminded for the 
$k-\omega$ Shear Stress Transport (SST) turbulence model. After introducing the experimental database used in this study (section III), section IV is devoted to the presentation of the modeling strategy and the derivation of roughness/blowing corrections adapted to the $k-\omega$ SST model. Finally, a large section is dedicated to the analysis and the assessment of the new model.

\section{SURFACE ROUGHNESS AND BLOWING ANALYSIS}

To study mean flow properties of the inner region of turbulent boundary layers, it is convenient to introduce dimensionless variables:

$$
\begin{gathered}
u^{+}=\frac{u}{u_{\tau}}, \\
y^{+}=\frac{\rho_{w} y u_{\tau}}{\mu_{w}},
\end{gathered}
$$

where $u$ and $y$ denote the longitudinal velocity and the distance normal to the surface. The subscript $w$ stands for wall quantities. The friction velocity $u_{\tau}$ is defined from the wall shear stress $\tau_{w}$ and the wall fluid density $\rho_{w}$ as

$$
u_{\tau}=\sqrt{\frac{\tau_{w}}{\rho_{w}}} .
$$

For compressible turbulent boundary layers over smooth wall without pressure gradient, dimensionless velocity profiles follow the standard law of the wall using Van Driest transformation in the inertial region

$$
u^{*+}=\int_{0}^{u^{+}} \sqrt{\frac{\rho}{\rho_{w}}} d u^{+}=\frac{1}{\kappa} \ln y^{+}+C,
$$

where $\kappa$ and $C$ are the von Kármán constant and the integration constant, respectively. In eddy viscosity RANS turbulence models, the wall shear stress $\tau_{w}$ and the wall heat flux $\phi_{w}$ are defined as

$$
\begin{gathered}
\tau_{w}=\left.\left(\mu+\mu_{t}\right) \frac{\partial u}{\partial y}\right|_{w}, \\
\phi_{w}=-\left.\left(\lambda+\lambda_{t}\right) \frac{\partial T}{\partial y}\right|_{w},
\end{gathered}
$$

where $\mu_{t}$ and $\lambda_{t}$ are the eddy viscosity and the eddy thermal conductivity. Obviously, on smooth wall without transpiration, $\mu_{t}$ and $\lambda_{t}$ vanish at the wall. To avoid additional transport equations for $\lambda_{t}$, the Reynolds analogy is usually employed in the context of turbulent boundary layers by introducing the turbulent Prandtl number

$$
P r_{t}=\frac{\mu_{t} C_{p}}{\lambda_{t}}
$$

where $C_{p}$ is the specific heat capacity of the fluid.

Most experimental results are presented in terms of skin friction coefficients and Stanton numbers where wall shear stress and heat flux are scaled by the boundary layer edge conditions

$$
\begin{gathered}
\frac{C_{f}}{2}=\frac{\tau_{w}}{\rho_{e} u_{e}^{2}}, \\
S t=\frac{\phi_{w}}{\rho_{e} u_{e}\left(h_{w}-h_{i e}\right)},
\end{gathered}
$$

where $h_{i}$ is the total enthalpy. The subscript $e$ refers to boundary layer edge conditions. It should be noted that the definitions of the skin friction coefficient and the Stanton number may differ between studies. In the presence of shock waves, it is common practice to use freestream flow conditions as in Holden experiments ${ }^{36}$. Unless otherwise specified, definitions of Eq. (8) and (9) will be used in section $\mathrm{V}$ for the assessment of the present model.

\section{A. Surface roughness effects}

Surface roughness modeling in a RANS context is largely based on Nikuradse's work ${ }^{8}$. Nikuradse highlighted three regimes of roughness:

- The aerodynamically smooth regime where the height of the roughness elements is lower than the viscous sublayer thickness. Thus, the surface roughness has no influence on skin friction.

- The fully rough regime where the viscous sublayer disappears due to the presence of higher roughness elements. Consequently, pressure drag dominates viscous shear stress and surface roughness effects become independent of the Reynolds number (i.e. viscosity).

- The transition regime, characterized by the contribution of both viscous and pressure drag to skin friction.

Nikuradse pointed out that the similarity parameter characterizing the effect of surface roughness in the fully rough regime is the dimensionless roughness height

$$
k_{s}^{+}=\frac{\rho_{w} k_{s} u_{\tau}}{\mu_{w}} .
$$

The roughness element height $k_{s}$ is also known as the equivalent sand grain height, a concept introduced by Schlichting ${ }^{9}$.

Moreover, Nikuradse showed that the velocity profile is described by the standard law of the wall but shifted downwards due to the surface roughness effect. By introducing the velocity shift $\Delta u_{r}^{+}$, also called the roughness function, the law of the wall can be expressed as

$$
u^{*+}=\frac{1}{\kappa} \ln y^{+}+C-\Delta u_{r}^{+} .
$$


The preservation of the logarithmic behavior reflects the validity of Townsend's hypothesis ${ }^{5}$ which assumes the independence of the outer region from wall conditions. Nikuradse proposed the following relation for the velocity shift:

$$
\begin{gathered}
\Delta u_{r}^{+}=\frac{1}{\kappa} \ln k_{s}^{+}+C-b_{1}-b_{2} \ln k_{s}^{+}, \\
\kappa=0.4 \quad C=5.5,
\end{gathered}
$$

where

$$
\begin{array}{rll}
k_{s}^{+}<3.5 & b_{1}=5.5 & b_{2}=1 / \kappa, \\
3.5 \leq k_{s}^{+}<7 & b_{1}=6.59 & b_{2}=1.52, \\
7 \leq k_{s}^{+}<14 & b_{1}=9.58 & b_{2}=0 \\
14 \leq k_{s}^{+}<68 & b_{1}=11.5 & b_{2}=-0.7, \\
68 \leq k_{s}^{+} & b_{1}=8.48 & b_{2}=0 .
\end{array}
$$

As viscous drag plays an important role in the transition regime, the behavior of surface roughness in this regime is not universal and depends on the roughness geometry considered. According to Colebrook's study ${ }^{40}$, Grigson ${ }^{41}$ proposed the following transitional behavior:

$$
\begin{gathered}
\Delta u_{r}^{+}=\frac{1}{\kappa} \ln \left(1+\frac{k_{s}^{+}}{\exp [\kappa(8.5-C)]}\right), \\
\kappa=0.41 \quad C=5.25 .
\end{gathered}
$$

It is worth noticing that Nikuradse's correlation and Colebrook's correlation are equivalent in the fully rough regime (i.e. $k_{s}^{+}>68$ ) with the same value of the von Kármán constant $\kappa$ and the integration constant $C$. In the transition regime, Nikuradse's correlation tends to provide lower roughness effects than Colebrook's correlation.

The universal behavior exhibited by Nikuradse experiments in the fully rough regime is the cornerstone of the equivalent sand grain approach. Based on the similarity parameter $k_{s}^{+}$, Schlichting ${ }^{9}$ proposed to assimilate any roughness geometry to an equivalent sand grain height which yields the same skin friction increase in Nikuradse's fully rough regime. This approach is widely used in industrial context. Nevertheless, its application to the transition regime is tricky since the dimensionless roughness height is no longer the only similarity parameter in this regime. For that reason, both Nikuradse's correlation and Colebrook's correlation may be employed in the transition regime in order to predict an envelop of possible surface roughness effects.

In RANS turbulence models, the logarithmic shift velocity $\Delta u_{r}^{+}$can be introduced by artificially increasing the turbulent eddy viscosity in the wall vicinity. Several authors ${ }^{14-16}$ developed roughness corrections adapted to the $k-\omega$ SST model. We retained the recent roughness correction of Aupoix ${ }^{16}$. This correction consists in a modification of turbulent kinetic energy and specific dissipation rate wall conditions. Based on Nikuradse's correlation, the wall conditions write

$$
\begin{aligned}
k_{w}^{+} & =\max \left(0, k_{0}^{+}\right), \\
k_{0}^{+} & =\frac{1}{\sqrt{\beta^{*}}} \tanh \left[\left(\frac{\ln \frac{k_{s}^{+}}{30}}{\ln 8}+0.5\left[1-\tanh \frac{k_{s}^{+}}{100}\right]\right) \tanh \frac{k_{s}^{+}}{75}\right], \\
\omega_{w}^{+} & =\frac{400000}{k_{s}^{+4}}\left(\tanh \frac{10000}{3 k_{s}^{+3}}\right)^{-1}+\frac{70}{k_{s}^{+}}\left[1-\exp \left(-\frac{k_{s}^{+}}{300}\right)\right] .
\end{aligned}
$$

Heat transfers are overpredicted by assuming a constant turbulent Prandtl number since the Reynolds analogy is no longer valid with the equivalent sand grain approach. By relying on the discrete element method, Aupoix ${ }^{17}$ proposed a correction on the turbulent Prandtl number in the following form:

$$
\operatorname{Pr}_{t}=\operatorname{Pr}_{t-s m o o t h}+\Delta \operatorname{Pr}_{t}\left(\Delta u_{r}^{+}, S_{\text {corr }}, \frac{y}{k}\right),
$$

where $k$ and $S_{\text {corr }}$ denote the mean roughness height and the corrected wetted surface ratio, respectively. The corrected wetted surface derives from the mean elevation concept introduced by McClain et al. ${ }^{42}$ for dense roughness geometries. Its evaluation is performed in the same way as for the wetted surface but from the elevated surface. Further information are available in Aupoix's study $^{17}$. The turbulent Prandtl number is increased by adding the shift $\Delta P r_{t}$. Thus, Aupoix's correction lowers the eddy thermal conductivity and the predicted heat transfer.

\section{B. Wall blowing effects}

The study of turbulent boundary layers with blowing is a subject of strong interest for researchers in view of their potential aeronautic applications (drag and heat transfer reduction). The recent review proposed by Kornilov ${ }^{18}$ provides a good compilation of experimental and numerical investigations on this topic. The analysis of blowing effects requires the introduction of some blowing parameters:

- the blowing rate $F=\frac{\rho_{w} v_{w}}{\rho_{e} u_{e}}$,

- the explicit blowing coefficients $B_{f}=2 F / C_{f 0}$ and $B_{h}=F / S t_{0}$,

- the dimensionless blowing velocity $v_{w}^{+}=v_{w} / u_{\tau}$.

where the subscript 0 refers to the non-blowing cases. The velocity $v$ denotes the wall-normal velocity. The first two parameters are usually employed to correlate skin friction coefficients and Stanton numbers using Couette flow theory. Implicit forms of the blowing coefficients can be introduced as well. The dimensionless blowing velocity appears in the description of the law of the wall 
and is a key parameter of blowing corrections for RANS turbulence models.

Under Couette flow assumption, the streamwise momentum equation can be expressed as

$$
\rho_{w} v_{w} \frac{\partial u}{\partial y}=\frac{\partial}{\partial y}\left(\left[\mu+\mu_{t}\right] \frac{\partial u}{\partial y}\right) .
$$

Integrating Eq. (17) with respect to y in the inner region yields

$$
\tau_{w}=\tau-\rho_{w} v_{w} u .
$$

Consequently, blowing results in a strong wall shear stress decrease caused by the mean wall normal convection. The description of the law of the wall derives from the Prandtl's mixing length theory. Eq. (18) can be integrated, leading to the so-called logarithmic law of the wall for compressible transpired turbulent boundary layers

$$
u^{* *+}=\int_{0}^{u^{+}} \frac{\sqrt{\frac{\rho}{\rho_{w}}}}{\sqrt{1+v_{w}^{+} u^{+}}} d u^{+}=\frac{1}{\kappa} \ln y^{+}+C\left(v_{w}^{+}\right),
$$

where the integration constant $C$ is a function of the dimensionless blowing velocity. Note that for incompressible turbulent boundary layers, Eq. (19) reduces to Stevenson's law of the wall. Originally, Stevenson ${ }^{21}$ suggested that the intercept constant $C$ is independent of the blowing velocity. Since, the decrease of $C$ with blowing was observed by many authors and is commonly accepted. This effect comes from the enhancement of turbulent motions in the wall vicinity caused by blowing. Similarly to surface roughness effect, the increase of the Reynolds stress near the wall results in a velocity shift on Stevenson's law.

Generally, the dependence of the constant $C$ is poorly reproduced by RANS turbulence models in their standard form. Therefore, RANS turbulence models without blowing corrections tend to underpredict skin friction coefficients and Stanton numbers for medium and high blowing rates. By relying on Andersen's study ${ }^{26}$, Wilcox ${ }^{13}$ proposed a correction to account for blowing effects in $k-\omega$ based models. This correction consists in a reduction of the specific dissipation rate wall condition

$$
\omega_{w}^{+}=\frac{20}{v_{w}^{+}\left(1+5 v_{w}^{+}\right)} .
$$

It should be noted that coefficient 20 is changed to 25 in the revised version of the model ${ }^{43}$. Wilcox's blowing correction has the same effect as roughness corrections. The idea is to enhance the turbulent eddy viscosity in the wall region in order to shift downwards the velocity profile and reproduce the dependency of the intercept constant $C$ on the dimensionless blowing velocity $v_{w}^{+}$.

By nature, roughness and blowing corrections cannot be employed together for $k-\omega$ based models since they play on the same wall boundary conditions. As will be seen in section IV, the only use of roughness corrections are not sufficient to predict the correct velocity shift in the case of combined effect of surface roughness and blowing. That's why, a new model based on existing roughness corrections is proposed in this paper.

\section{EXPERIMENTAL DATABASE}

First of all, a detailed description of the available experimental data is made in this section. As noted in the introduction, only few studies investigated the combined effects of surface roughness and blowing. All the experimental configurations are summarized in Table I by specifying the measurements performed. The first two experiments (Moffat and $\mathrm{Kays}^{44}$ and Andersen et al. ${ }^{26}$ ) do not include roughness effects but are used with the aim of extending the proposed model to the aerodynamically smooth regime.

At Stanford University, an extensive series of experimental works on heat and mass transfer for transpired turbulent boundary layers were performed. The first experiment of Moffat and Kays ${ }^{44}$ and Andersen's work ${ }^{26}$ constitute recognized references for turbulent boundary layers over smooth wall with uniform blowing. Moffat and Kays experiments include temperature profiles and Stanton numbers while velocity profiles and skin friction were measured in Andersen's work.

Healzer et al. ${ }^{31}$ were the firsts to investigate the effect of roughness on transpired turbulent boundary layers. Both skin friction coefficient and Stanton number were measured along the test surface. The experiments were conducted at uniform free stream velocities of 10, 27, 42, 58 and $74 \mathrm{~m} . \mathrm{s}^{-1}$ leading to a roughness Reynolds number $k_{s}^{+}$range from 20 to 200 without blowing. Therefore, the experimental conditions are sufficient to provide a large range of roughness effects from the transition regime to the fully rough regime. The porous test surface is composed of copper balls of $1.27 \mathrm{~mm}$ diameter, brazed together in the most dense arrangement. According to Schlichting experiments ${ }^{9}$, the recommended value of the equivalent sand grain height is 0.625 times the ball diameter. Nonetheless, in order to predict the exact skin friction of the non-blowing configurations, a slight modification of the recommended equivalent sand grain height is proposed. The idea is to model the effect of blowing starting with a correct prediction of the surface roughness. As the use of the recommended value proposed by Schlichting leads to a slight error on the skin friction of non-blowing configurations, a different value was taken to avoid unwanted skin friction shifts on blowing configurations. In the present work, a value of 0.56 times the ball diameter was taken, yielding an equivalent sand grain height of $0.711 \mathrm{~mm}$ (close to Schlichting's recommended value of $0.794 \mathrm{~mm}$ ). It should be noted that the correct equivalent sand grain depends on the selected roughness correlation since Nikuradse's law and Colebrook's law slightly differ in the fully rough regime. 


\begin{tabular}{|c|c|c|c|c|c|c|c|c|c|}
\hline \multirow{2}{*}{ Authors } & \multicolumn{4}{|c|}{ Configuration } & \multicolumn{5}{|c|}{ Measurements } \\
\hline & Geometry & Roughness & $F\left(\times 10^{3}\right)$ & $M_{e}$ & $C_{f}$ & $S t$ & $u$ & $T$ & Turbulence \\
\hline Moffat and Kays ${ }^{44}$ & Flat plate & $x$ & $0-10$ & - & $x$ & $\checkmark$ & $x$ & $\checkmark$ & $x$ \\
\hline Andersen et al. ${ }^{26}$ & Flat plate & $x$ & $0-8$ & - & $\checkmark$ & $x$ & $\checkmark$ & $x$ & $\checkmark$ \\
\hline Healzer et $a l .{ }^{31}$ & Flat plate & Densely packed spheres & $0-4$ & - & $\checkmark$ & $\checkmark$ & $\checkmark$ & $\checkmark$ & $x$ \\
\hline Pimenta et $a l^{45}$ & Flat plate & Densely packed spheres & $0-4$ & - & $\checkmark$ & $\checkmark$ & $\checkmark$ & $\checkmark$ & $\checkmark$ \\
\hline Coleman et al. ${ }^{46}$ & Flat plate & Densely packed spheres & $0-4$ & - & $\checkmark$ & $x$ & $\checkmark$ & $x$ & $\checkmark$ \\
\hline Miller et al. ${ }^{35}$ & Channel & Sinusoidal roughness & $0-1.6$ & - & $\checkmark$ & $x$ & $\checkmark$ & $x$ & $\checkmark$ \\
\hline Schetz and Nerney ${ }^{34}$ & Axisymmetric body & Porous surface & $0-4$ & - & $\checkmark$ & $x$ & $\checkmark$ & $x$ & $x$ \\
\hline Voisinet $^{24}$ & Flat plate & Roughness screen & $0-3$ & 3 & $\checkmark$ & $x$ & $\checkmark$ & $\checkmark$ & $x$ \\
\hline Holden $^{36}$ & Cone $10.5^{\circ}$ & Hemispherical & $0-9$ & $7.5-8$ & $\checkmark$ & $\checkmark$ & $x$ & $x$ & $x$ \\
\hline
\end{tabular}

TABLE I: Experimental studies of combined effects of blowing and surface roughness.

The wall to free stream temperature difference was maintained between $10 \mathrm{~K}$ and $20 \mathrm{~K}$ to ensure constant flow properties. The wetted corrected surface ratio used for the thermal correction is estimated at 1.142 by considering a roughness geometry of densely packed hemispheres. This value was successful used by Aupoix ${ }^{17}$ for the prediction of Healzer ${ }^{31}$ and Coleman ${ }^{46}$ experiments on nonblowing cases. Among the five test section velocities, only the three highest are considered in this study to prevent the issue of laminar-turbulent transition. For these configurations, the boundary layer transition is complete near the beginning of the test plate. Stanton numbers are determined from an energy balance while skin friction coefficients are estimated using the integral momentum equation. Consequently, skin friction presents a higher degree of scattering than Stanton numbers. Healzer suggested that the uncertainty in Stanton numbers is around $\pm 10^{-4}$. For several reasons, the estimation of the skin friction uncertainty is difficult. Healzer concluded that experimental uncertainties in skin friction probably exceed $\pm 10 \%$ with blowing. We will only keep the uncertainty of $\pm 10^{-4}$ in Stanton numbers for the validation of the present model in section $\mathrm{V}$.

Pimenta et al. ${ }^{45}$ extended Healzer experiments to turbulent shear stress measurements using hot wire. To improve the determination of the skin friction, Pimenta used Andersen's shear stress method. For this reason, only Pimenta's velocity profiles in wall units are considered in section $\mathrm{V}$ and not those measured by Healzer. The effect of blowing were studied at uniform free stream velocity of $27 \mathrm{~m} \cdot \mathrm{s}^{-1}$. As the porous test surface is identical in both experiments of Healzer and Pimenta, the same set of surface roughness parameters is chosen. Thereafter, Coleman et al. ${ }^{46}$ concluded the series of experimental works on Healzer's configurations by investigating the effects of favorable pressure gradients.

Voisinet $^{24}$ studied the combined effects of surface roughness and blowing on skin friction under adiabatic wall condition at Mach number of 2.9. Direct measurements of the wall shear stress were performed using a skin friction gage. In addition to skin friction, bound- ary layer profile surveys are also given. All experimental data are available in Voisinet's technical report ${ }^{47}$. The measurements were obtained at fixed streamwise location and not along the test plate. Voisinet experiments were conducted at various Reynolds numbers per meter from 0.1 to $3 \times 10^{6} \mathrm{~m}^{-1}$ in order to provide a wide range of surface roughness and blowing effects. Three mass flow rates were tested for blowing $(0.015,0.049$ and $0.146 \mathrm{~kg} \cdot \mathrm{m}^{-2} \cdot \mathrm{s}^{-1}$ ). The porous surface is composed of multilayer composites in which surface roughness effects are provided by bonding an additional screen mesh. Three roughness screen heights were considered $(0.1,0.33$ and $1.25 \mathrm{~mm}$ ). As noted by Voisinet, roughness screens are problematic regarding geometrical modeling. Consequently, an equivalent sand grain height cannot be determined with classical roughness correlations ${ }^{10-12}$. By comparing velocity profiles for non-blowing configurations, an equivalent sand grain height of 0.625 times the roughness height is proposed in the present study. This value appears to be well adapted to roughness correction based on Nikuradse's law (see section V).

Holden $^{36,48-51}$ conducted a series of three experimental studies of separate and combined effects of surface roughness and blowing in hypersonic turbulent boundary layers over a $10.5^{\circ}$ sharp slender cone. However, the simulation of Holden's configurations raises many problems. The experimental uncertainties seem to be very significant since Holden's data present a high degree of scattering. The validity of Townsend's hypothesis cannot be proved as the ratio of the roughness height to the boundary layer thickness is generally more than $10 \%$. Moreover, for hypersonic cold-wall cases (i.e. $T_{w} / T_{f}<1$ ), compressibility corrections $^{52-54}$ of turbulence models must be used in order to improve skin friction and heat transfer predictions. These corrections are based on the turbulent Mach number $M_{t}$ as an indicator of the compressibility of the turbulence. Nevertheless, the use of roughness corrections (equivalent sand grain approach) leads to an artificial increase of the turbulent Mach number in the inner region. Consequently, compressibility corrections and roughness corrections interfere in this region yielding questionable 


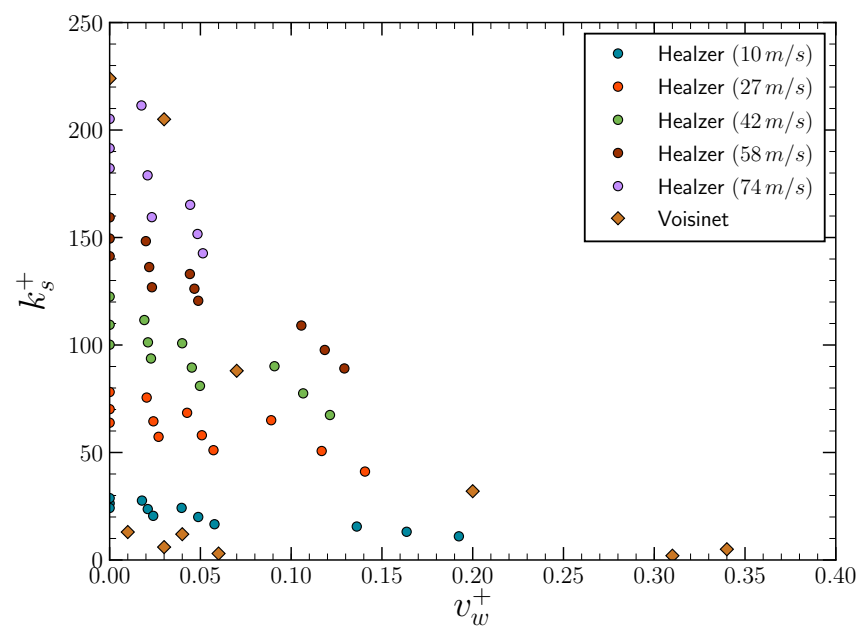

FIG. 1: Roughness and blowing experimental domains

skin friction predictions. For these reasons, Holden experiments are not in the scope of the present paper for the assessment of the proposed model.

The last two experiments (Schetz and Nerney ${ }^{34}$ and Miller et $a l .{ }^{35}$ ) are also not considered in the present work for several reasons. First, the velocity profiles measured by Schetz and Nerney are not in agreement with Stevenson's law as the slope of their profiles are not altered. Second, experimental results obtained by Miller showed that uniform blowing increases drag friction and, thus, enhances surface roughness effects. Furthermore, the addition of blowing resulted in the reduction of near wall turbulent motions, contrasting with FIK analysis ${ }^{20}$. Consequently, these experiments disagreed with the common accepted analysis of blowing effects.

The domain covered by the retained experiments in the $\left(k_{s}^{+}, v_{w}^{+}\right)$plane is presented in Fig. 1 . The present experimental database appears to be representative of a large range of surface roughness effects. As the friction velocity increases with roughness effects, experiments usually provide low dimensionless blowing velocities $v_{w}^{+}$but sufficient to observe significant blowing effects. Conversely, when blowing effects are important, the roughness Reynolds numbers $k_{s}^{+}$are generally small since the friction velocity decreases. Nevertheless, there is a lack of experimental data with medium combined effects of surface roughness and blowing. Therefore, additional experiments are welcome to improve the present model and justify its applicability to a large panel of configurations.

\section{MODELING STRATEGY}

\section{A. Combined effects of roughness and blowing}

The present modeling relies upon the characterization of the velocity profile in the inertial region and the identification of the separate contributions of blowing and surface roughness on the velocity shift $\Delta u^{+}$of the logarithmic law. Various authors ${ }^{24,37}$ pointed out that the logarithmic behavior of the velocity profile in transpired turbulent boundary layers over rough wall is altered in the following general form:

$$
u^{* *+}=\frac{1}{\kappa} \ln y^{+}+C-\left.\Delta u^{+}\right|_{\text {app }}\left(k_{s}^{+}, v_{w}^{+}\right) .
$$

This form reflects the overlap of independent blowing and roughness effects. On the one hand, the slope of the velocity profile is modified by the addition of blowing, leading to Stevenson's law of the wall. On the other hand, the logarithmic law obtained in similarity variables (i.e. $u^{* *+}$ ) is shifted downwards by the drag increasing contribution of the surface roughness. As blowing affects the mixing process between roughness elements, the apparent velocity shift $\left.\Delta u^{+}\right|_{a p p}$ depends not only on the dimensionless equivalent sand grain height $k_{s}^{+}$but also on the dimensionless blowing velocity $v_{w}^{+}$. The overlap of independent effects can also be seen as the result of the validity of Townsend's hypothesis for surface roughness effects. The interactions between roughness and blowing remain confined to the inner region and, thus, Stevenson's law of the wall is preserved in the inertial region.

By nature, Stevenson's law is obtained by solving RANS equations, independently of the selected turbulence model as they are all calibrated to recover the mixing length behavior in the inertial region. Therefore, the prediction of the combined effects of surface roughness and blowing involves obtaining the correct wall shear stress only (i.e. the correct apparent velocity shift). However, it is worth noticing that Stevenson's law depends on the dimensionless blowing velocity and, thus, depends on the wall shear stress. Consequently, the slope of the velocity profiles is overestimated by standard RANS turbulence models which tend to provide too weak wall shear stress without blowing corrections (see section V).

The apparent velocity shift can be decomposed in the following manner:

$$
\left.\Delta u^{+}\right|_{\text {app }}\left(k_{s}^{+}, v_{w}^{+}\right)=\Delta u_{r}^{+}\left(k_{s}^{+}\right)+\Delta u_{r b}^{+}\left(k_{s}^{+}, v_{w}^{+}\right) .
$$

The first term represents the roughness contribution given by the common roughness correlations (Nikuradse's law or Colebrook's law). The second term depicts the effect of the interaction between roughness elements and blowing on the skin friction. The interest of this decomposition is to rely on existing roughness corrections. As surface roughness effects exhibit a logarithmic behavior in the fully rough regime, an apparent equivalent sand grain can be defined in such a way that both contributions emerge in the velocity shift.

The estimation of the velocity shift $\Delta u_{r b}^{+}$was performed on the basis of Healzer's skin friction and the velocity profiles measured by Pimenta and Voisinet in the fully rough regime. It was found that the velocity shift $\Delta u_{r b}^{+}$is independent of the equivalent sand grain height in this regime. Consequently, the logarithmic behavior of the surface roughness effects is preserved in the 


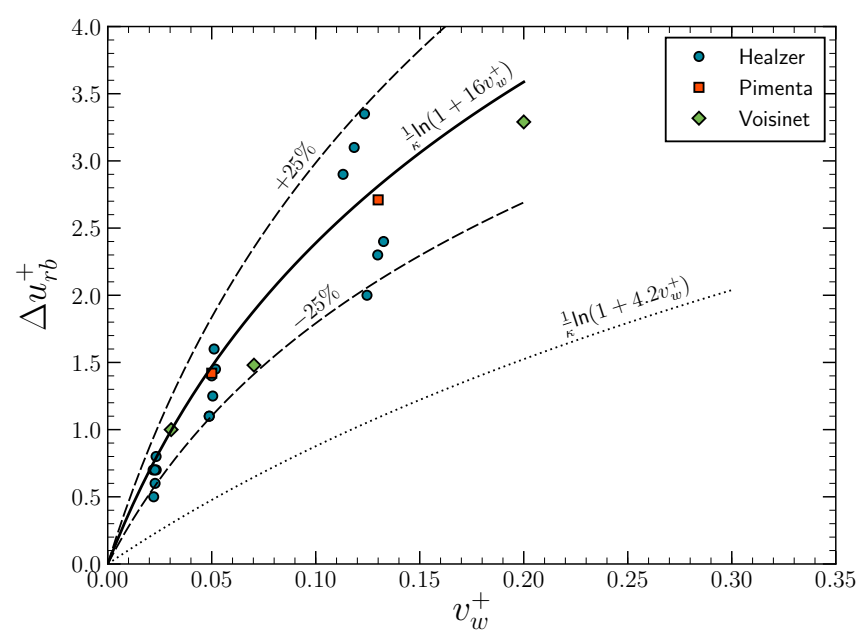

FIG. 2: Estimation of the velocity shift $\Delta u_{r b}^{+}$in the fully rough regime. Solid line (-) corresponds to the proposed correlation. Dotted line (.....) represents the velocity shift induces by blowing over smooth walls

fully rough regime with blowing. The resulting velocity shifts $\Delta u_{r b}^{+}$are plotted in Fig. 2 with respect to the dimensionless blowing velocity. Inherent in the measurement method, a high degree of scattering is observed with Healzer's data. The following relationship is proposed to correlate the velocity shift $\Delta u_{r b}^{+}$in the fully rough regime:

$$
\Delta u_{r b}^{+}=\frac{1}{\kappa} \ln \left(1+16 v_{w}^{+}\right) .
$$

The proposed correlation is built in such a manner that it is consistent with the logarithmic behavior of roughness correlations (Eq. (12) and Eq. (14)). Substituting each contribution into Eq. (22), we obtain

$$
\left.\Delta u^{+}\right|_{a p p}=\frac{1}{\kappa} \ln k_{s}^{+}+C-8.5+\frac{1}{\kappa} \ln \left(1+16 v_{w}^{+}\right) .
$$

As a result, the present modeling in the fully rough regime reduces to

$$
\left.k_{s}^{+}\right|_{\text {app }}=k_{s}^{+}\left(1+16 v_{w}^{+}\right) .
$$

The blowing correction proposed by Healzer and used in his numerical computations using Van Driest mixing length model ${ }^{55}$ is then recovered.

Extension of the present model to the aerodynamically smooth regime was made using the velocity profiles measured by Andersen. The following correlation is introduced to represent the velocity shift induced by blowing over smooth walls:

$$
\Delta u_{r b}^{+}=\frac{1}{\kappa} \ln \left(1+4.2 v_{w}^{+}\right) .
$$

It should be noted that the velocity shift $\Delta u_{r b}^{+}$over smooth wall is lower than that over rough wall (see
Fig. 2). That's why, the combination of independent turbulent corrections (blowing and roughness) cannot predict the combined effects of surface roughness and blowing accurately.

For the same reasons as for surface roughness modeling, the transition regime is complex to characterize using the equivalent sand grain approach. There are a variety of possible transitional behaviors depending on the considered surface geometry. Furthermore, the estimation of the velocity shift $\Delta u_{r b}^{+}$based on experimental data cannot be performed in a simple manner since the roughness contribution $\Delta u_{r}^{+}$does not generally follow the behavior given by the common roughness correlations in this regime. In the light of the above comments, a general form is proposed to predict combined effects of surface roughness and blowing in the transition regime

$$
\Delta u_{r b}^{+}\left(k_{s}^{+}, v_{w}^{+}\right)=\frac{1}{\kappa} \ln \left(1+f\left(k_{s}^{+}\right) v_{w}^{+}\right),
$$

where

$$
f=5.9\left[1+\tanh \left(\frac{k_{s}^{+}-k_{s, \text { trans }}^{+}}{\Delta_{k_{s}^{+}}}\right)\right]+4.2 .
$$

The function $f$ ranges from 4.2 to 16 to ensure the transition. By chance, the surface roughness used by Voisinet behaves like Nikuradse's law in the transition regime as will be explained in section V. Hence, the transition function $f$ can be calibrated using Voisinet's velocity profiles and Nikuradse's correlation. The following transition function is proposed:

$$
f\left(k_{s}^{+}\right)=5.9\left[1+\tanh \left(k_{s}^{+}-7\right)\right]+4.2 .
$$

The present modeling remains independent of the selected RANS turbulence model. However, it should be applied properly since the response of RANS turbulence models to wall blowing conditions differs considerably. For instance, the Spalart-Allmaras turbulence model ${ }^{29}$ behaves correctly in the presence of blowing and, thus, doesn't need additional blowing corrections. The reason is that this turbulence model provides naturally an accurate velocity shift $\Delta u_{r b}^{+}$for blowing configurations over smooth walls. Therefore, in order to apply the present modeling to the Spalart-Allmaras model, the velocity shift $\Delta u_{r b}^{+}$over smooth walls (i.e. Eq. (26)) must be removed from the apparent velocity shift $\left.\Delta u^{+}\right|_{a p p}$. The advantage of $k-\omega$ based models is that the predicted velocity profiles $u^{* *+}$ for blowing configurations tend to collapse in a unique logarithmic law as emphasized by Chedevergne and Marchenay ${ }^{30}$. In other words, the predicted velocity profiles do not exhibit additional velocity shifts caused by blowing. For this reason, the present modeling can be applied directly to $k-\omega$ based models.

Finally, using Colebrook's correlation, the modeling of combined effects of surface roughness and blowing adapted to $k-\omega$ based models reads

$$
\left.k_{s}^{+}\right|_{\text {app }}=k_{s}^{+}\left(1+f v_{w}^{+}\right)+f v_{w}^{+} e^{\kappa(8.5-C)} .
$$


The present modeling using Nikuradse's correlation is given by

$$
\left.k_{s}^{+}\right|_{\text {app }}=\max \left(k_{1}, k_{2}\right),
$$

where

$$
\begin{aligned}
& k_{1}=k_{s}^{+}\left(1+f v_{w}^{+}\right), \\
& k_{2}=\exp \left[\frac{\kappa\left(b_{1}-C\right)}{1-\kappa b_{2}}\right]\left(1+f v_{w}^{+}\right)^{1 /\left(1-\kappa b_{2}\right)} .
\end{aligned}
$$

As the slope of Nikuradse's correlation is defined by part, additional modifications were made to preserve the continuity of the apparent equivalent sand grain. Thus, the predicted velocity shift is slightly different to that given by Eq. (27) in the transition regime.

The apparent velocity shift $\left.\Delta u^{+}\right|_{\text {app }}$ obtained from the modification of the equivalent sand grain is illustrated in Fig. $3 \mathrm{a}$ and $3 \mathrm{~b}$ for four different blowing velocities. The first plot represents the apparent Nikuradse's law using Eq. (31) while the second plot shows the apparent Colebrook's law using Eq. (30). As noted previously, in the fully rough regime the logarithmic behavior of the velocity shift is preserved with the addition of blowing but shifted upwards by the contribution of $\Delta u_{r b}^{+}$. The aerodynamically smooth regime is characterized by the independence of the present model on the equivalent sand grain height $k_{s}^{+}$. Therefore, the apparent velocity shift reduces to the contribution of blowing over smooth walls. The present model is equivalent to the Wilcox's correction (Eq. (20)) in this regime. It can be noticed that the range of the transition regime is significantly decreased while increasing the blowing velocity. For example, the proposed model based on Nikuradse's law predicts a full transition at $k_{s}^{+}=20$ for $v_{w}^{+}=0.2$ while standard transition is around 70 . This property is consistent with Healzer and Pimenta experiments in which the fully rough regime persists to small values of $k_{s}^{+}$with no evidence of the transition regime. It can be explained considering the effect of blowing on the viscous sublayer. The transition regime is correlated with the presence of the viscous sublayer which contributes to the skin friction. However, blowing exhibits a destructive effect on the viscous sublayer similar to surface roughness effects. Therefore, the transition to the fully rough regime is promoted by increasing blowing velocity. One may argue that the range of the transition regime looks very short for high blowing velocities, suggesting non physical behaviors. Nevertheless, the proposed transition function $f$ seems to be representative of the behavior of Voisinet's experiment surface roughness as will be discussed in section V.

The final step is the extension of the present modeling to thermal effects. As mentioned in section II, the equivalent sand grain approach tends to overestimate the wall heat flux by using the Reynolds analogy ${ }^{17}$. The problem derives from the artificial enhancement of the turbulent motions in the near wall region. Conversely, blowing corrections preserve the Reynolds analogy ${ }^{56}$. For that reason, the thermal behavior of transpired turbulent boundary layers over rough wall is complex to describe and model. Due to the lack of experimental data focused on this topic, quite a few assumptions have been examined. First, we assumed the validity of Aupoix's thermal correction (Eq. (16)) in the presence of blowing with the aim of predicting the correct thermal roughness behavior. Second, only the velocity shift is modified to account for blowing effects in the thermal correction. The remaining parameters (i.e. $y / k$ and $S_{\text {corr }}$ ) are assumed to be unaffected by blowing since they are geometrical parameters. As the present modeling concerns the characterization of the velocity shift, it seems natural to modify the velocity shift $\Delta u_{r}^{+}$in Aupoix's thermal correction. The apparent velocity shift $\left.\Delta u^{+}\right|_{\text {app }}$ is a natural choice but it does not tend to zero when surface roughness effects become negligible. Therefore, a modification of the transition function $f$ must be made to ensure the canceling of the thermal correction for blowing configuration over smooth walls. As will be seen in section V, a good prediction is obtained using the apparent velocity shift $\left.\Delta u^{+}\right|_{\text {app }}$ for Healzer experiments. Finally, the turbulent Prandtl number correction in the presence of combined effects of surface roughness and blowing reads

$$
P r_{t}=P r_{t-s m o o t h}+\Delta P r_{t}\left(\left.\Delta u^{+}\right|_{a p p}, S_{c o r r}, \frac{y}{k}\right),
$$

where the transition function $f$ is altered as follows:

$$
f_{T}=8.0\left[1+\tanh \left(k_{s}^{+}-7\right)\right] .
$$

This modification is only used for the determination of the apparent velocity shift $\left.\Delta u^{+}\right|_{a p p}$ in the turbulent Prandtl number correction. The transition function $f_{T}$ is equivalent to the previous one in the fully rough regime. However, it tends to zero in the aerodynamically smooth regime to ensure the Reynolds analogy (i.e. $\left.\left.\Delta u^{+}\right|_{\text {app }}=0\right)$.

\section{B. Numerical considerations}

The assessment of the present correction was performed using the ONERA two-dimensional boundary layer code CLICET ${ }^{57}$. Self-adaptive grid procedures based on the estimation of the wall normal gradients of each variable ensure the grid convergence. The height of the first grid cell center is fixed in such a way that the reduced height $y^{+}$is around 0.1 . The initialization of the computations is carried out by employing self-similar solutions to generate boundary layer profiles. This code was extensively used to predict surface roughness and blowing effects in turbulent boundary layers (Aupoix ${ }^{16,17}$ and Chedevergne and Marchenay ${ }^{30}$ ).

Menter $k-\omega$ SST model ${ }^{58}$ in conservation form is written as follows:

$$
\mu_{t}=\frac{\rho a_{1} k}{\max \left(a_{1} \omega ; \Omega F_{2}\right)},
$$




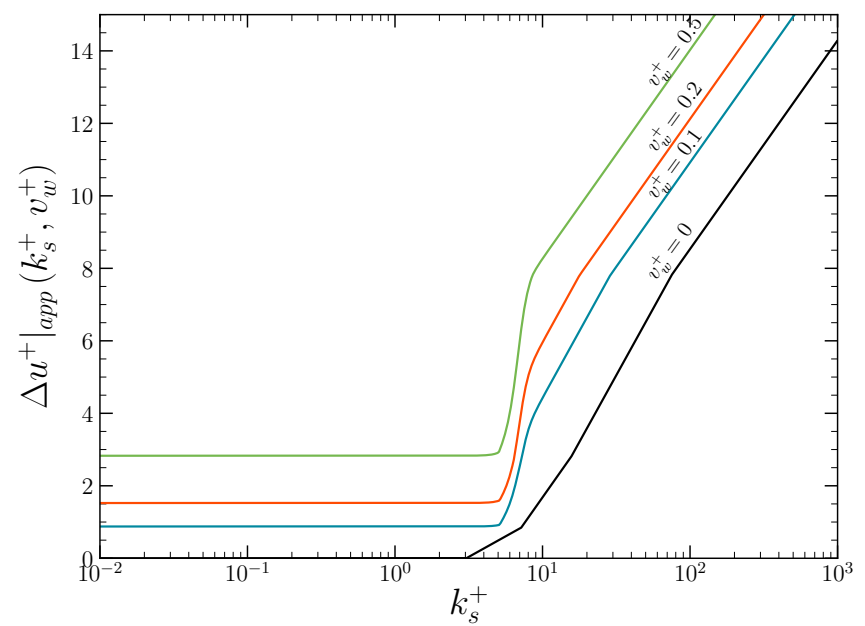

(a)

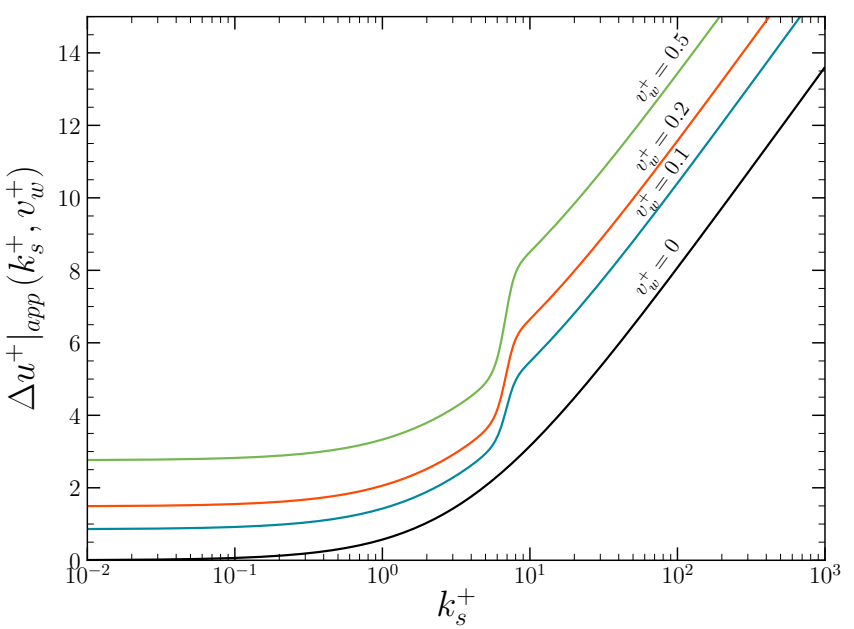

(b)

FIG. 3: Estimation of the velocity shift $\left.\Delta u^{+}\right|_{\text {app }}$ given by the roughness correlations for various $v_{w}^{+}$. (a) Apparent Nikuradse's law. (b) Apparent Colebrook's law.

$$
\begin{aligned}
\frac{\partial(\rho k)}{\partial t}+\frac{\partial\left(\rho u_{j} k\right)}{\partial x_{j}}= & P_{k}-\beta^{*} \rho k \omega+\frac{\partial}{\partial x_{j}}\left[\left(\mu+\sigma_{k} \mu_{t}\right) \frac{\partial k}{\partial x_{j}}\right] \\
\frac{\partial(\rho \omega)}{\partial t}+\frac{\partial\left(\rho u_{j} \omega\right)}{\partial x_{j}}= & \gamma \frac{\rho}{\mu_{t}} P_{k}-\beta \rho \omega^{2}+\frac{\partial}{\partial x_{j}}\left[\left(\mu+\sigma_{\omega} \mu_{t}\right) \frac{\partial \omega}{\partial x_{j}}\right] \\
& +2\left(1-F_{1}\right) \rho \frac{\sigma_{\omega_{2}}}{\omega} \frac{\partial k}{\partial x_{j}} \frac{\partial \omega}{\partial x_{j}}
\end{aligned}
$$

where the production of the turbulent kinetic energy $P_{k}$ is defined using Boussinesq relation

$$
P_{k}=-\overline{\rho u_{i}^{\prime \prime} u_{j}^{\prime \prime}} \frac{\partial u_{i}}{\partial x_{j}}=\left[\mu_{t}\left(2 s_{i j}-\frac{2}{3} \frac{\partial u_{l}}{\partial x_{l}} \delta_{i j}\right)-\frac{2}{3} \rho k \delta_{i j}\right] \frac{\partial u_{i}}{\partial x_{j}} .
$$

The blending function $F_{1}$ is designed to ensure the transition between Wilcox $k-\omega$ model in the near wall region (i.e. $F_{1}=1$ ) and the $k-\varepsilon$ model in the wake region of the boundary layer (i.e. $F_{1}=0$ ). A full description of the model parameters can be found in Menter's paper ${ }^{58}$.

As a reminder, the proposed model consists in using the $k-\omega$ SST turbulence model and roughness corrections but with the modification of the equivalent sand grain height (i.e. Eq. (30) or Eq. (31)) and the modification of the turbulent Prandtl number correction (i.e. Eq. (34)). These modifications were integrated directly into the boundary layer code CLICET to perform the simulations.

\section{RESULTS AND DISCUSSION}

The objective of this section is twofold. First, the predictions of existing corrections are illustrated with the aim of demonstrating the interest of a specific modeling of combined effects of roughness and blowing. To that end, the predictions of the $k-\omega$ SST turbulence model (dashed lines ---) in combination with roughness corrections and wall blowing conditions (without additional specific corrections) are presented in all figures. Then, the proposed model (solid lines — ) is assessed against experimental data for each configuration described in section III. Nevertheless, as the roughness laws are equivalent in the fully rough regime, we chose to only present the results obtained using Nikuradse's correlation. Moreover, the interest of Nikuradse's correlation is that it predicts accurately the behavior of Voisinet experiment surface roughness in the transition regime.

\section{A. Stanford experiments}

The first considered experiments are those conducted at Stanford University (Moffat and Kays ${ }^{44}$, Andersen et $a l .{ }^{26}$, Healzer et $a l .{ }^{31}$, Pimenta et $a l .{ }^{45}$ and Coleman et $\left.a l .{ }^{46}\right)$. Andersen and Moffat experiments are used to assess the proposed model in the aerodynamically smooth regime (i.e. for $k_{s}^{+}=0$ ). Five blowing rates were investigated $\left(F=0,1,2,4\right.$ and $\left.8 \times 10^{-3}\right)$. Skin friction coefficients and Stanton numbers are illustrated in Fig. 4a and 4b against the Reynolds number $R e_{x}$. Skin friction results for the highest blowing rate case $\left(F=8 \times 10^{-3}\right)$ are not presented due to excessive experimental uncertainties (near separation behaviors). As expected, computations without blowing corrections underestimate skin friction coefficients and Stanton numbers. The problem comes from the underestimation of near wall turbulent motions as mentioned in section II. Using Wilcox's correction (Eq. (20)), the predictions of skin friction are improved and experimental results are well reproduced. Similar results are obtained with the proposed correction (Eq. (31) for $k_{s}^{+}=0$ ), which proves the efficiency of the correction 


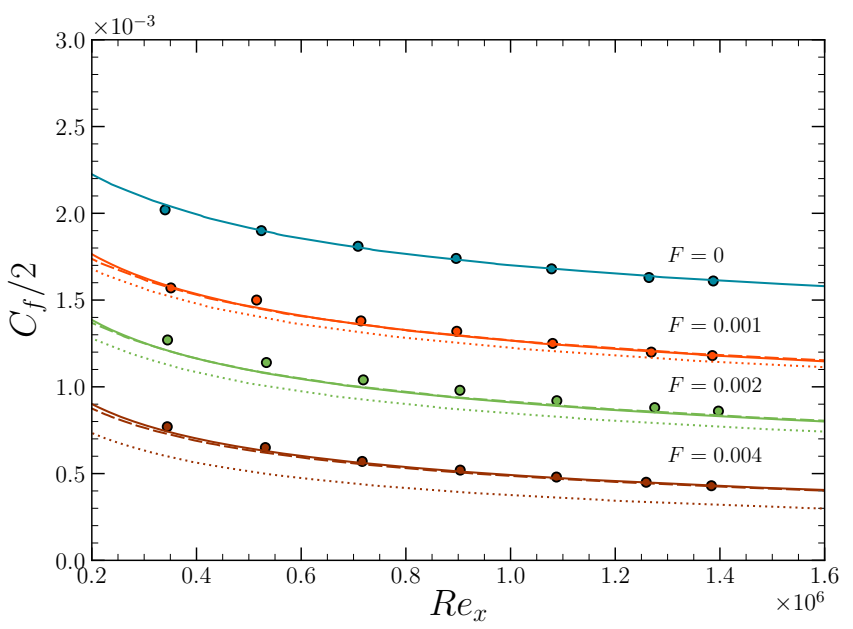

(a)

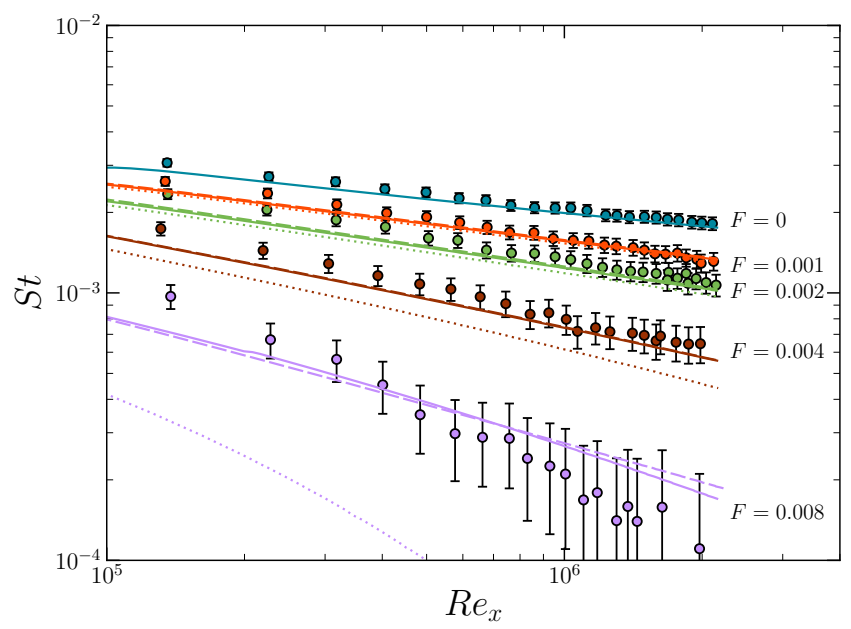

(b)

FIG. 4: Turbulent boundary layers over smooth walls for several blowing rates $F$. Symbols represent experimental data: (a) Andersen et al. and (b) Moffat and Kays. Dotted lines (.....) are results obtained without blowing correction, dashed lines (- - ) using Wilcox's correction and solid lines (- ) with the proposed correction. (a) Skin friction coefficients. (b) Stanton numbers.

in the smooth regime. Furthermore, Stanton number predictions are in fair agreement with Moffat and Kays experiments without modifying the turbulent Prandtl number. This reflects the validity of the Reynolds analogy for blowing cases over smooth walls.

Healzer experiments $\left(u_{e}=42 \mathrm{~m} . \mathrm{s}^{-1}\right)$ are shown in Fig. $5 \mathrm{a}$ and $5 \mathrm{~b}$ in terms of skin friction coefficients and Stanton numbers for several blowing rates $F$. Skin friction coefficients and Stanton numbers are plotted against the Reynolds number based, respectively, on the momentum thickness $\theta$ and the enthalpy thickness $\Delta_{T}$ to avoid problems related to the boundary layer origin. First, we note a good agreement between the predictions using Nikuradse based roughness correction and Healzer's non-blowing data (i.e. $F=0$ ). For this configuration, the equivalent sand grain height $k_{s}^{+}$is about 100 at the end of the plate and, thus, lies in the fully rough regime. The use of Aupoix's thermal correction leads to better predictions of Stanton numbers by reducing the eddy thermal conductivity in the wall vicinity. In the presence of blowing, skin friction obtained with Nikuradse based roughness correction (-- -) are clearly underestimated. For the higher blowing case (i.e. $F=0.004$ ), the difference rises up to around $60 \%$ at the end of the plate. Similar differences are also observed for Stanton numbers but remain small compared to skin friction predictions. For the same case, the error rises up to around 30\%. The difference between skin friction and Stanton number predictions derives from the effect of the roughness thermal correction which tends to limit the impact of skin friction levels on Stanton numbers. More specifically, the effect of the thermal correction increases with higher skin friction as it depends on the velocity shift $\Delta u_{r}^{+}$. Therefore, the differences observed on Stanton numbers are necessarily lower than those noted on skin friction. The efficiency of the proposed correction (-) based on Nikuradse apparent law (i.e. Eq. (31)) is visible in Fig. 5a and 5b. By adding the contribution $\Delta u_{r b}^{+}$in the apparent velocity shift of the logarithmic law, skin friction levels are increased in comparison with uncorrected ones. We note a really good agreement with Healzer experiments. The skin friction error decreases to less than $10 \%$ at the end of the plate for each blowing case. For the higher blowing case, the roughness Reynolds number $k_{s}^{+}$is reduced to about 70 while the apparent roughness Reynolds number $\left.k_{s}^{+}\right|_{\text {app }}$ is about 190 . Consequently, the term $\Delta u_{r b}^{+}$contributes to $20 \%$ of the apparent velocity shift $\left.\Delta u^{+}\right|_{\text {app }}$. Similar results are obtained for Stanton numbers. As skin friction levels are enhanced by the proposed correction, Stanton numbers are naturally improved. For the sake of clarity, higher free stream velocity cases $\left(u_{e}=58\right.$ and $74 \mathrm{~m} . \mathrm{s}^{-1}$ ) are not presented in this paper. Results are very similar and in agreement with Healzer's data.

Comparisons with Pimenta experiments are illustrated in Fig. 6a, 6b and 6c. Similar results are obtained for skin friction. Without specific blowing/roughness corrections, skin friction levels are largely underestimated by the $k-\omega$ SST turbulence model. Using the proposed correction, skin friction predictions are improved and experimental data are well reproduced. Velocity profiles in similarity variables (see Eq. (19) for incompressible cases) are plotted in Fig. 6b. Pimenta's profiles are those measured at $x=1.88 \mathrm{~m}$ (i.e. 74 inch) and the comparison is performed at the same Reynolds number $R e_{\theta}$. In similarity variables, the velocity profiles exhibit a logarithmic behavior in the inertial region, equivalent to the standard law of the wall but shifted downwards. As discussed in section IV, the apparent velocity shift depends on both 


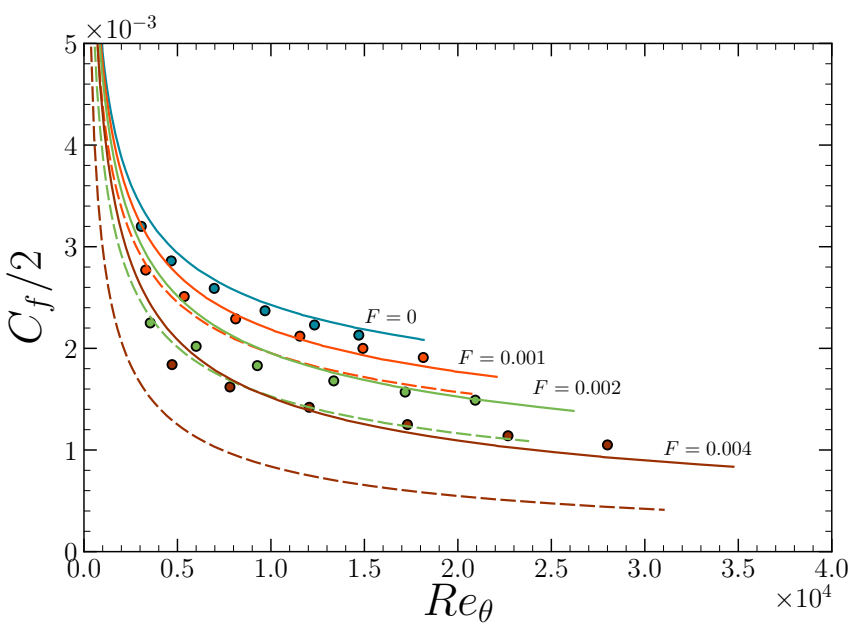

(a)

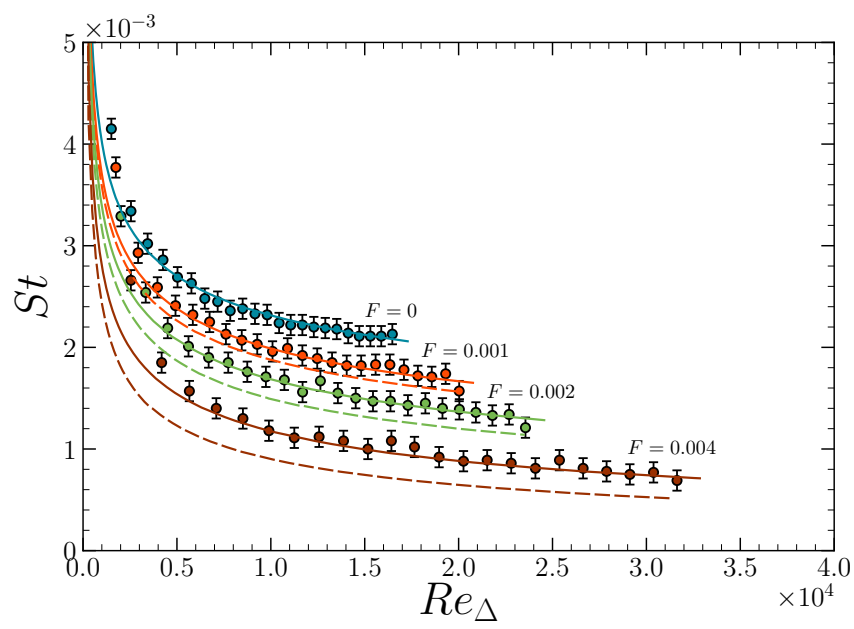

(b)

FIG. 5: Turbulent boundary layers over rough walls for several blowing rates F. Symbols represent Healzer's data $\left(u_{e}=42 \mathrm{~m} \cdot \mathrm{s}^{-1}\right)$. Dashed lines (-- $)$ are results obtained with the roughness correction based on Nikuradse's correlation and solid lines (-) using the proposed correction. (a) Skin friction coefficients. (b) Stanton numbers.

parameters $k_{s}^{+}$and $v_{w}^{+}$. One may note that the apparent velocity shift of Pimenta's profiles is increased by blowing. Since roughness Reynolds numbers $k_{s}^{+}$are reduced by adding blowing, standard roughness corrections (- - ) can not reproduce the enhancement of the velocity shift and, thus, overestimate the levels of the velocity profiles. The use of the proposed correction improves considerably the prediction of the apparent velocity shift by adding an additional contribution: $\Delta u_{r b}^{+}$. Then, the transition to wall variables is performed naturally by the turbulence model as shown in Fig. 6c. In any case, Stevenson's law of the wall is recovered. However, since the friction velocity $u_{\tau}$ (i.e. $\Delta u_{\text {app }}^{+}$) is underestimated using the standard roughness correction, the velocity profiles in wall variables (i.e. $u^{+}$) exhibit too stiff slopes.

To finish exploring Stanford experiments, Coleman's configurations are considered in Fig. 7. We retained three cases as illustrated in Fig. 7a. The first two cases are characterized by the same favorable pressure gradient but with or without uniform blowing. The last one is defined by the presence of steps in blowing and wall temperature. Stanton numbers are illustrated in Fig. 7b against the streamwise coordinate $x$. Without blowing, the agreement between predictions and Coleman's data is very good and proves the efficiency of the roughness correction (Eq. (15)) and Aupoix's thermal correction (Eq. (16)) in presence of favorable pressure gradients. As expected, the roughness correction underestimates Stanton numbers on blowing cases. The difference rises up to around $20 \%$ at $x=1 \mathrm{~m}$. Again, the proposed correction (Eq. (31)) improves the predictions by increasing Stanton numbers. Furthermore, the presence of steps in $F$ and $T_{w}$ for the last case is well reproduced by the model. Velocity profiles compared at the same position $x=1$ $\mathrm{m}$ are plotted in Fig. 7c. The velocity profiles for the last case are not measured by Coleman and, thus, are not present. We note an excellent agreement with Coleman's data using the new roughness/blowing correction. As the correction increases the shear stress in the inner region, velocity profiles are decreased compared with the uncorrected ones. Moreover, one may notice that the boundary layer thickness is underestimated with the standard roughness correction, which is not the case using the proposed correction. In the light of the previous comments, the strategy adopted seems perfectly effective for incompressible turbulent boundary layers.

\section{B. Voisinet's data}

Voisinet experiments concerned supersonic turbulent boundary layers over rough wall at Mach 2.9. Among all velocity profiles measured by Voisinet, we chose to present only a few that characterize the three roughness regimes (aerodynamically smooth, transition and fully rough). Results are very similar for the other profiles. As before with Pimenta's profiles, the comparison is performed at the same Reynolds number $R e_{\theta}$. First, we consider velocity profiles in the fully rough regime. Results are plotted in Fig. 8, in wall variables (8a) and in similarity variables (8b). As expected, the predicted profile for the non-blowing case (i.e. $k_{s}^{+}=225$ ) is in good agreement with measurements since the equivalent sand grain height was chosen to reproduce Voisinet experiment surface roughness. In presence of blowing, the slope of the velocity profiles is altered, following a bilogarithmic form (i.e. $u^{* *+}$ ). The predicted profiles are very similar to those obtained in incompressible turbulent boundary layers. Without a specific correction, the turbulence model tends to underestimate the skin fric- 


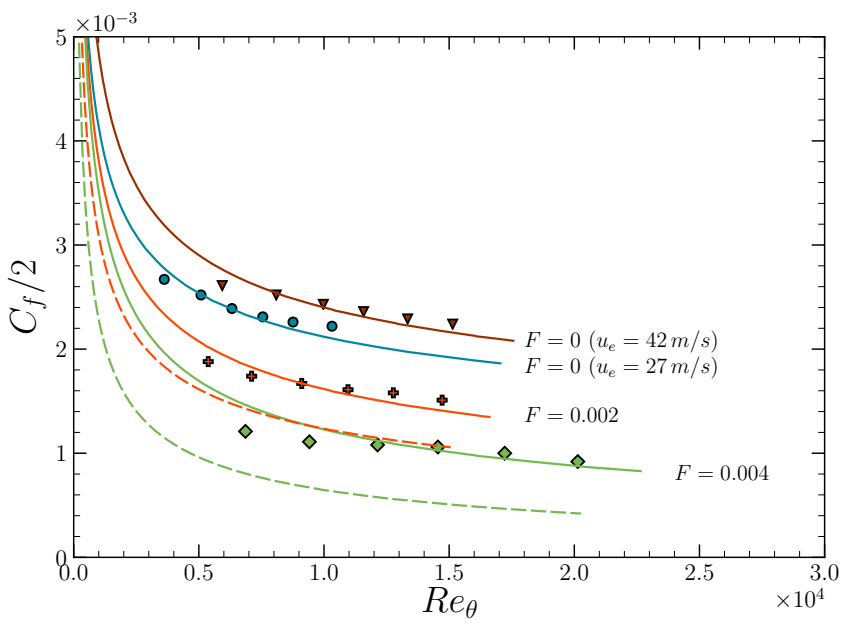

(a)

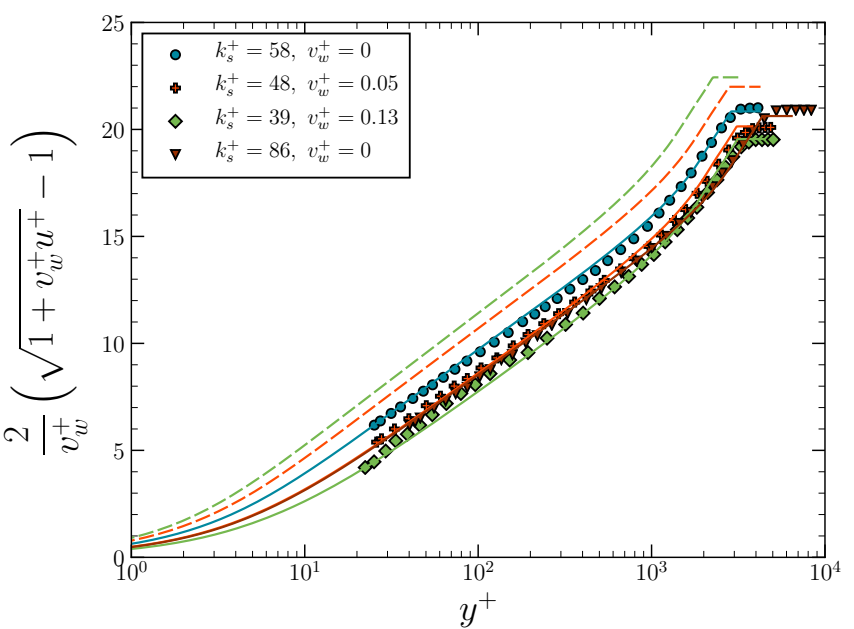

(b)

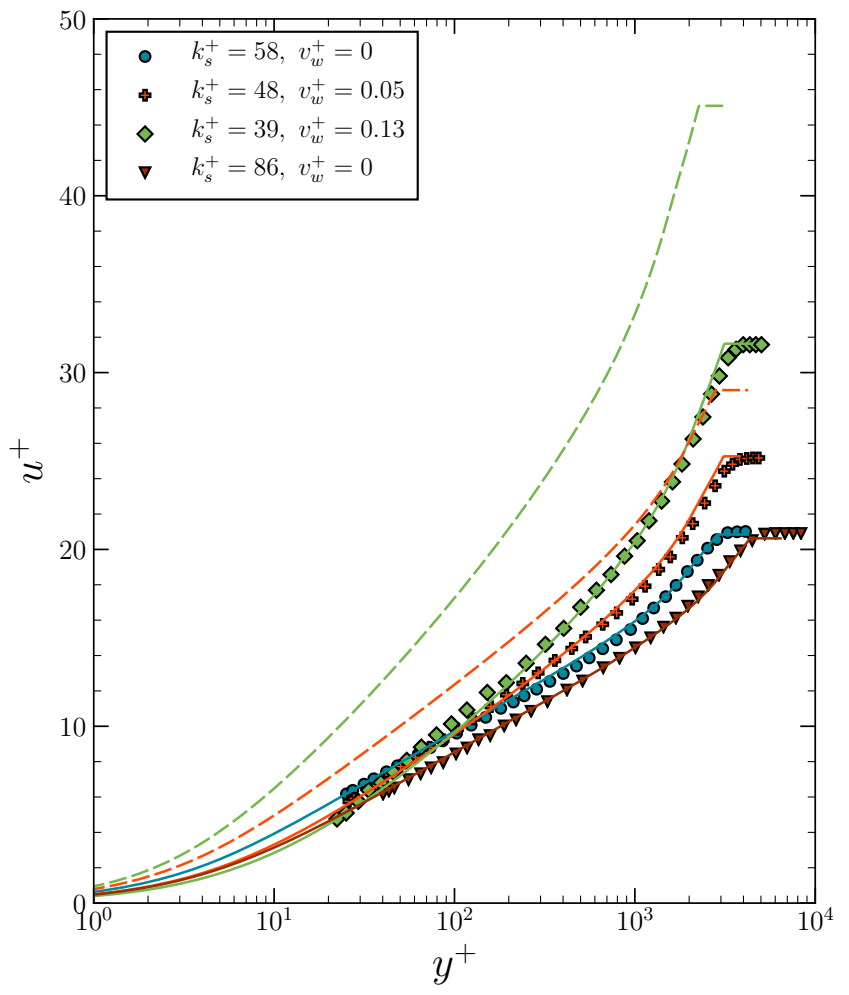

(c)

FIG. 6: Turbulent boundary layers over rough walls for several blowing rates F. Symbols represent Pimenta's data. Dashed lines (- - ) are results obtained with the roughness correction based on Nikuradse's correlation and solid lines (-) using the proposed correction. (a) Skin friction coefficients. (b) Velocity profiles in similarity variables. (c) Velocity profiles in wall variables.

tion and, thus, overestimates the slope of the velocity profiles. The difference is clearly visible for the highest blowing rate case (i.e. $v_{w}^{+}=0.2$ ) where the uncorrected profile is close to boundary layer separation. Again, the use of the proposed correction leads to better results. One may note a slight underestimation of the velocity profile for the highest blowing rate case. It appears to be related to an incorrect prediction of the wake of the turbulent boundary layer and not to the prediction of the apparent velocity shift $\left.\Delta u^{+}\right|_{\text {app }}$. When increasing $v_{w}^{+}$, the inlet conditions in Voisinet experiments change and the velocity profiles in the wake region are altered, as if a slight adverse pressure gradient was introduced. Despite this small restriction, the apparent velocity shifts are well 


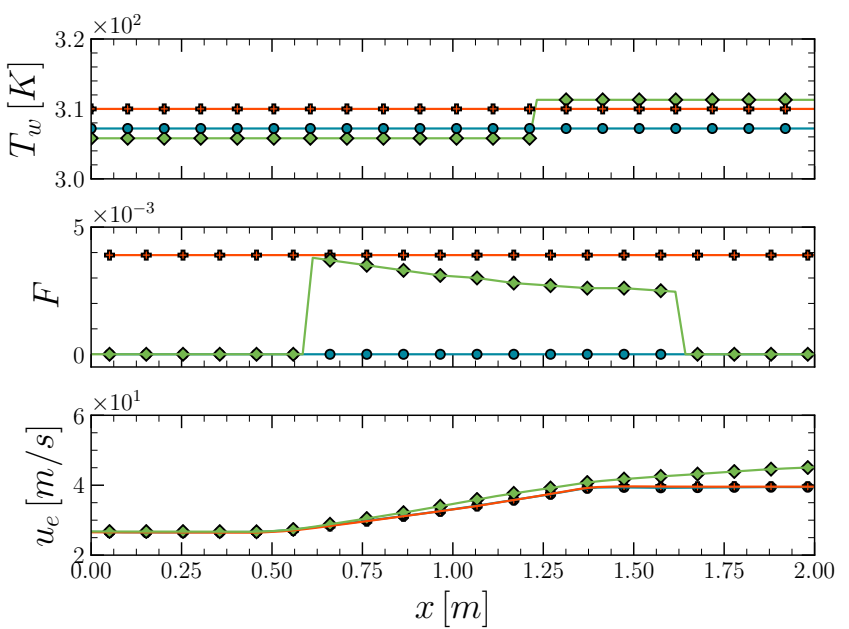

(a)

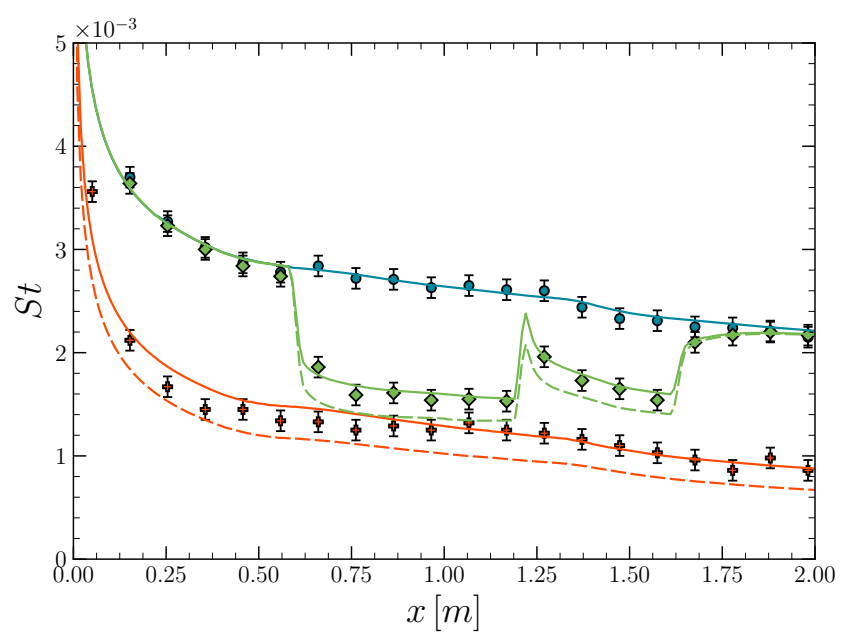

(b)

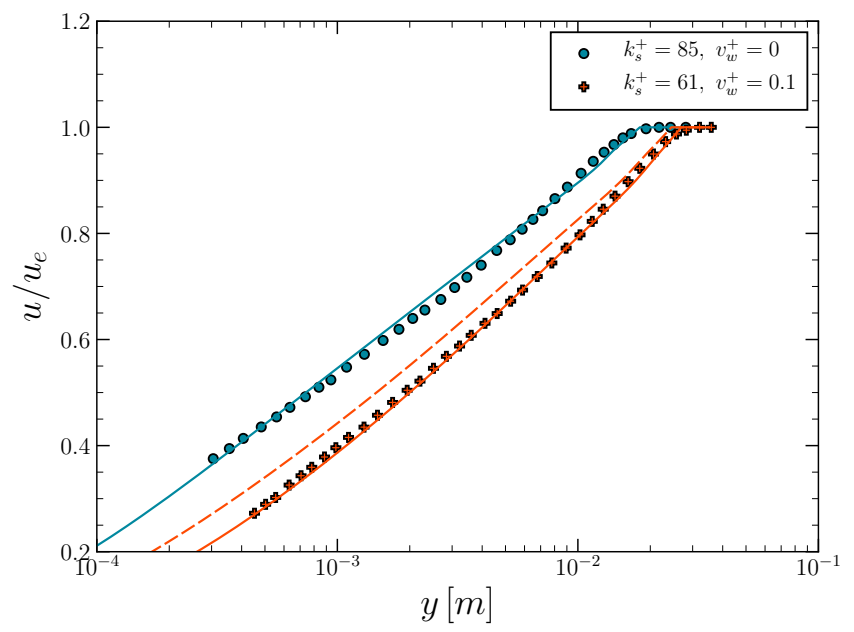

(c)

FIG. 7: Turbulent boundary layers over rough walls subjected to favorable pressure gradients. Symbols represent

Coleman's data. Dashed lines (- - ) are results obtained with the roughness correction based on Nikuradse's correlation and solid lines (-) using the proposed correction. (a) Experimental conditions. (b) Stanton numbers. (c) Velocity profiles.

reproduced by the proposed correction as we can see in Fig. 8b. Again, using the roughness correction only, the apparent velocity shift is considerably underestimated. The difference for the highest blowing rate case rises to about $60 \%$ in the inertial region while using the proposed correction, the difference decreases to less than $10 \%$.

Velocity profiles in the transition regime are illustrated in Fig. 9a and 9b. The first plot concerns non-blowing cases while the second illustrates velocity profiles for several blowing rates. As mentioned in section IV, Nikuradse's law accurately reproduces the velocity shifts observed for Voisinet experiments surface roughness in the transition regime. That's why the transition function $f$ can be calibrated using Voisinet's profiles in this regime. The retained velocity profiles are plotted in Fig. 9b. As explained before, the slope of velocity profiles obtained with roughness corrections only tends to be overestimated with blowing. A large difference is found for the last two cases $\left(v_{w}^{+}=0.31\right.$ and 0.34$)$. As the roughness Reynolds numbers $k_{s}^{+}$are close to zero for this two cases, the roughness correction does not induce a velocity shift $\Delta u_{r}^{+}$on the log law. Consequently, the predicted velocity profiles are similar to those obtained over smooth wall without blowing corrections. The use of the proposed correction lead to better agreements with Voisinet's profiles since it relies on the apparent roughness Reynolds number $\left.k_{s}^{+}\right|_{\text {app }}$. Therefore, the proposed correction predicts an additional contribution $\Delta u_{r b}^{+}$but also a nonzero roughness contribution $\Delta u_{r}^{+}$. For the lowest blowing rates, results are similar with or without specific corrections as the dimensionless blowing velocity $v_{w}^{+}$is small. However, one may notice a slight improvement of the 


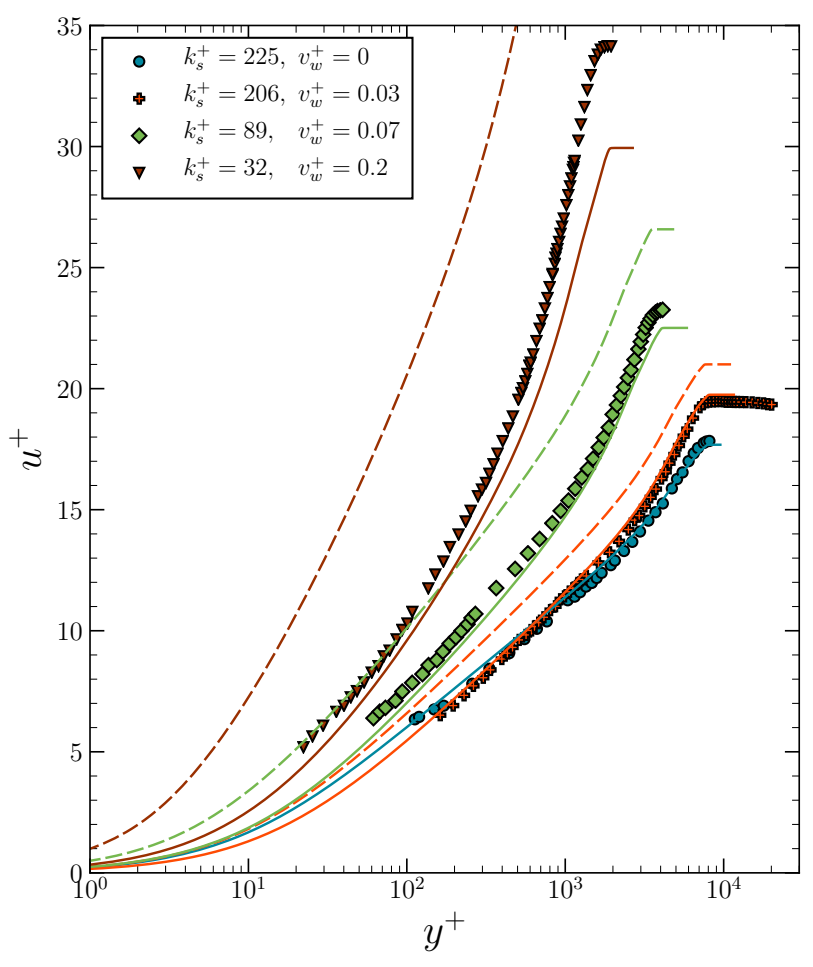

(a)

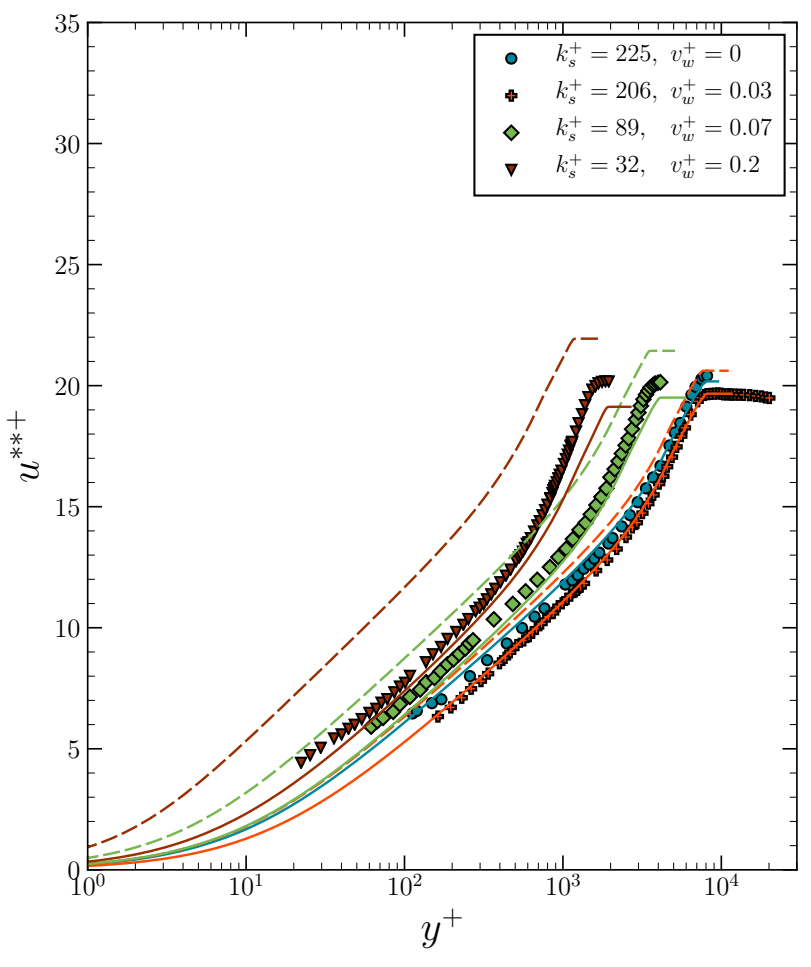

(b)

FIG. 8: Supersonic turbulent boundary layers over rough walls. Symbols represent Voisinet's data $\left(M_{e}=2.9\right)$. Dashed lines (-- ) are results obtained with the roughness correction based on Nikuradse's correlation and solid lines (-) using the proposed correction. (a) Velocity profiles in wall variables. (b) Velocity profiles in similarity variables.

predictions using the new blowing/roughness correction. Based on these results, the new modeling of combined effects of roughness and blowing seems to be effective for compressible turbulent boundary layers too.

\section{CONCLUSIONS}

A new modeling strategy adapted to RANS turbulence models was proposed to predict combined effects of surface roughness and blowing on incompressible and compressible turbulent boundary layers. First, the failure of existing models was pointed out using the $k-\omega$ SST turbulence model associated with roughness corrections. The latter tends to underestimate the wall shear stress and, thus, the wall heat flux in the presence of combined effects, leading to bad skin friction and Stanton number predictions. Moreover, as Stevenson's law depends on the wall shear stress, the slope of the velocity profiles is necessarily overestimated by existing models. The failure of these models derives from an inaccurate estimation of the apparent velocity shift of the log-law given by the roughness correction.

To improve boundary layer predictions, the proposed modeling is based on the characterization of the separate contributions of blowing and roughness effects on the apparent velocity shift $\left.\Delta u^{+}\right|_{\text {app }}$. Concretely, an additional contribution $\Delta u_{r b}^{+}$caused by blowing/roughness interactions was emphasized by analyzing a large set of experimental data. In order to integrate this contribution to standard roughness corrections, an apparent equivalent sand grain $\left.k_{s}^{+}\right|_{\text {app }}$ was derived. Two new blowing/roughness corrections based on Nikuradse and Colebrook roughness correlations were proposed, yielding different transitional behaviors. The extension of the present models to thermal effects was performed naturally by including the apparent velocity shift $\left.\Delta u^{+}\right|_{\text {app }}$ in Aupoix's thermal correction. The ability of the proposed models to predict combined effects of roughness and blowing was evidenced over a large range of roughness effects from the aerodynamically smooth regime to the fully rough regime by comparing results with experiments.

\section{DATA AVAILABILITY}

The data that support the findings of this study are available from the corresponding author upon reasonable request. 


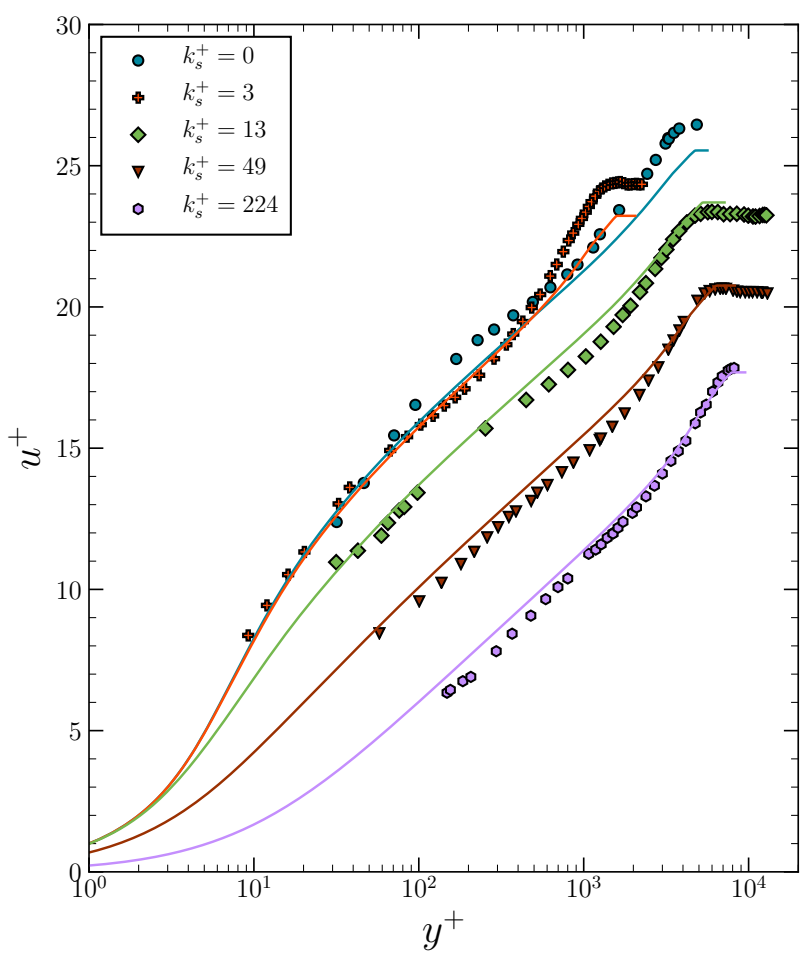

(a)

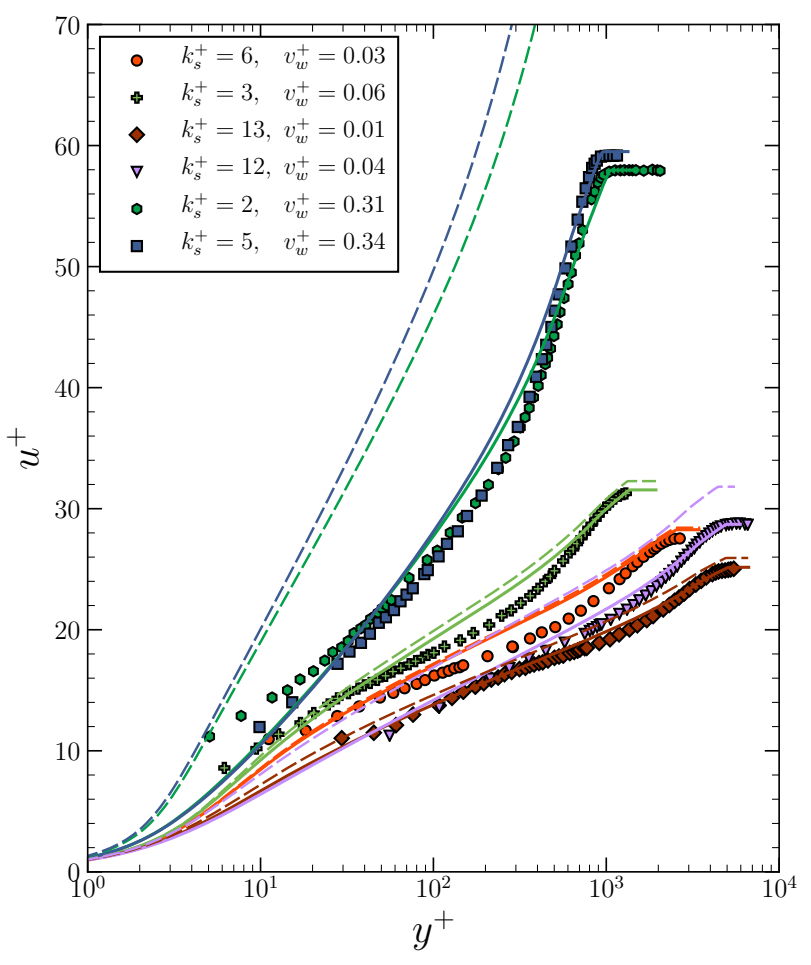

(b)

FIG. 9: Supersonic turbulent boundary layers over rough walls in the transition regime. Symbols represent Voisinet's velocity profiles $\left(M_{e}=2.9\right)$. Dashed lines (-- ) are results obtained with the roughness correction based on Nikuradse's correlation and solid lines (-) using the proposed correction. (a) Non-blowing cases. (b) Blowing

cases.

${ }^{1}$ G. L. Vignoles, J. Lachaud, Y. Aspa, and J.-M. Goyhénèche, "Ablation of carbon-based materials: Multiscale roughness modelling," Composites Science and Technology 69, 1470 - 1477 (2009), special Issue on the 12th European Conference on Composite Materials (ECCM12), organized by the European Society for Composite Materials (ESCM).

${ }^{2}$ P. M. Ligrani and R. J. Moffat, "Structure of transitionally rough and fully rough turbulent boundary layers," Journal of Fluid Mechanics 162, 69-98 (1986).

${ }^{3}$ K. A. Flack and M. P. Schultz, "Roughness effects on wallbounded turbulent flows," Physics of Fluids 26, 101305 (2014), https://doi.org/10.1063/1.4896280.

${ }^{4}$ J. P. Bons, "A Review of Surface Roughness Effects in Gas Turbines," Journal of Turbomachinery 132 (2010), $10.1115 / 1.3066315$.

${ }^{5}$ A. A. Townsend, The structure of turbulent shear flow (Cambridge University Press, 1976).

${ }^{6}$ K. A. Flack, M. P. Schultz, and J. S. Connelly, "Examination of a critical roughness height for outer layer similarity," Physics of Fluids 19, 095104 (2007), https://doi.org/10.1063/1.2757708.

7J. Jiménez, "Turbulent flows over rough walls," Annual Review of Fluid Mechanics 36, 173-196 (2004), https://doi.org/10.1146/annurev.fluid.36.050802.122103.

${ }^{8}$ J. Nikuradse, "Laws of flows in rough pipes," English Translation, NACA TM 1292 (1950)

${ }^{9} \mathrm{H}$. Schlichting, "Experimental investigation of the problem of surface roughness," Technical Memorandum No. 823 (NACA, 1936).

${ }^{10} \mathrm{R}$. B. Dirling Jr., "A method for computing rough wall heat transfer rates on reentry nosetips," in AIAA Paper No. 73-763 (AIAA 8th Thermophysics Conference, Palm Springs, California, 1973).
${ }^{11}$ A. Sigal and J. E. Danberg, "New correlation of roughness density effect on the turbulent boundary layer," AIAA journal 28, 554556 (1990).

${ }^{12}$ J. A. van Rij, B. J. Belnap, and P. M. Ligrani, "Analysis and Experiments on Three-Dimensional, Irregular Surface Roughness ," Journal of Fluids Engineering 124, 671-677 (2002).

${ }^{13} \mathrm{D}$. C. Wilcox, "Reassessment of the scale-determining equation for advanced turbulence models," AIAA Journal 26, 1299-1310 (1988).

${ }^{14}$ A. Hellsten and S. Laine, "Extension of $k-\omega$ shear-stress transport turbulence model for rough-wall flows," AIAA Journal 36, 1728-1729 (1998).

${ }^{15}$ T. Knopp, B. Eisfeld, and J. B. Calvo, "A new extension for $k-\omega$ turbulence models to account for wall roughness," International Journal of Heat and Fluid Flow 30, 54-65 (2009).

${ }^{16} \mathrm{~B}$. Aupoix, "Roughness corrections for the $k-\omega$ shear stress transport model: Status and proposals," Journal of Fluids Engineering, Transactions of the ASME 137 (2015), $10.1115 / 1.4028122$.

${ }^{17} \mathrm{~B}$. Aupoix, "Improved heat transfer predictions on rough surfaces," International Journal of Heat and Fluid Flow 56, 160-171 (2015).

${ }^{18} \mathrm{~V}$. Kornilov, "Current state and prospects of researches on the control of turbulent boundary layer by air blowing," Progress in Aerospace Sciences 76, 1-23 (2015).

${ }^{19} \mathrm{~J}$. Bellettre, F. Bataille, and A. Lallemand, "Prediction of thermal protection of walls by blowing with different fluids," International Journal of Thermal Sciences 38, $492-500$ (1999).

${ }^{20} \mathrm{~K}$. Fukagata, K. Iwamoto, and N. Kasagi, "Contribution of reynolds stress distribution to the skin friction in wall-bounded flows," Physics of Fluids 14, L73-L76 (2002). 
${ }^{21} \mathrm{~T}$. N. Stevenson, "A law of the wall for turbulent boundary layers with suction or injection," CoA, Report Aero No. 166 (The College of Aeronautics Cranfield, 1963).

${ }^{22}$ J. E. Danberg, "Characteristics of the turbulent boundary layer with heat and mass transfer at $\mathrm{M}=6.7$," Tech. Rep. (Aerodynamics research report No. 228, 1964).

${ }^{23}$ L. C. Squire, "A law of the wall for compressible turbulent boundary layers with air injection," Journal of Fluid Mechanics 37, 449-456 (1969).

${ }^{24}$ R. L. P. Voisinet, "Combined influence of roughness and mass transfer on turbulent skin friction at Mach 2.9," in 17th Aerospace Sciences Meeting (1979).

${ }^{25}$ I. Vigdorovich, "A law of the wall for turbulent boundary layers with suction: Stevenson's formula revisited," Physics of Fluids 28, 085102 (2016)

${ }^{26}$ P. S. Andersen, W. M. Kays, and R. J. Moffat, "Experimental results for the transpired turbulent boundary layer in an adverse pressure gradient," Journal of Fluid Mechanics 69, 353-375 (1975).

27 J. Bellettre, F. Bataille, and A. Lallemand, "A new approach for the study of turbulent boundary layers with blowing," International Journal of Heat and Mass Transfer 42, 2905-2920 (1999).

${ }^{28}$ R. Hink, V. Hannemann, and T. Eggers, "Extension of the spalart-allmaras one-equation turbulence model for effusive cooled rocket chambers," in 4th International Space Propulsion Conference (2014).

${ }^{29} \mathrm{P}$. Spalart and S. Allmaras, "A one-equation turbulence model for aerodynamic flows," in 30th aerospace sciences meeting and exhibit (1992) p. 439.

${ }^{30} \mathrm{~F}$. Chedevergne and Y. Marchenay, "Transpired turbulent boundary layers: a general strategy for rans turbulence models," Journal of Turbulence 20, 681-696 (2019).

${ }^{31}$ J. M. Healzer, R. J. Moffat, and W. M. Kays, "The turbulent boundary layer on a porous, rough plate: Experimental heat transfer with uniform blowing," in Thermophysics and Heat Transfer Conference (1974) p. 680.

${ }^{32}$ M. M. Pimenta, R. J. Moffat, and W. M. Kays, "The structure of a boundary layer on a rough wall with blowing and heat transfer," Journal of Heat Transfer 101, 193-198 (1979).

${ }^{33} \mathrm{H}$. Coleman, R. Moffat, and W. Kays, "Momentum and energy transport in the accelerated fully rough turbulent boundary layer," Stanford Univ. Report (1976).

${ }^{34}$ J. A. Schetz and B. Nerney, "Turbulent boundary layer with injection and surface roughness," AIAA Journal (1977).

${ }^{35}$ M. A. Miller, A. Martin, and S. C. C. Bailey, "Investigation of the scaling of roughness and blowing effects on turbulent channel flow," Experiments in Fluids 55, 1675 (2014).

${ }^{36} \mathrm{M}$. Holden, "Studies of surface roughness and blowing effects on hypersonic turbulent boundary layers over slender cones," in 27th Aerospace Sciences Meeting (1989).

${ }^{37}$ E. Mori, M. Quadrio, and K. Fukagata, "Turbulent drag reduction by uniform blowing over a two-dimensional roughness," Flow, Turbulence and Combustion 99, 765-785 (2017).

${ }^{38}$ G. H. Christoph and R. H. Pletcher, "Prediction of rough-wall skin friction and heat transfer," AIAA Journal 21, 509-515 (1983), https://doi.org/10.2514/3.8107.

39 J. Ilegbusi, "Proposal for wall function for friction and heat transfer in the presence of roughness and mass transfer," International Communications in Heat and Mass Transfer 11, 569 - 581 (1984).

${ }^{40}$ C. F. Colebrook, "Turbulent flow in pipes, with particular reference to the transition region between the smooth and rough pipe laws," Journal of the Institution of Civil engineers 11 (1939).

${ }^{41}$ C. Grigson, "Drag losses of new ships caused by hull finish," Journal of Ship Research 36, 182-196 (1992).

${ }^{42}$ S. T. McClain, S. P. Collins, B. K. Hodge, and J. P. Bons, "The Importance of the Mean Elevation in Predicting Skin Friction for Flow Over Closely Packed Surface Roughness," Journal of Fluids Engineering 128, 579-586 (2005).

${ }^{43}$ D. C. Wilcox, "Formulation of the kw turbulence model revisited," AIAA journal 46, 2823-2838 (2008).
${ }^{44}$ R. J. Moffat and W. M. Kays, "The turbulent boundary layer on a porous plate: Experimental heat transfer with uniform blowing and suction," International Journal of Heat and Mass Transfer 11, 1547-1566 (1968).

${ }^{45}$ M. M. Pimenta, R. J. Moffat, and W. M. Kays, "The turbulent boundary layer: An experimental study of the transport of momentum and heat with the effect of roughness," Report No. HMT-21 (Thermosciences Division, Department of Mechanical Engineering, Stanford Univ., 1975).

${ }^{46}$ H. W. Coleman, R. J. Moffat, and W. M. Kays, "The accelerated fully rough turbulent boundary layer," Journal of Fluid Mechanics 82, 507-528 (1977).

${ }^{47}$ R. L. P. Voisinet, "Influence of roughness and blowing on compressible turbulent boundary layer flow," Tech. Rep. (Naval Surface Weapons Center, White Oak Lab, 1979).

${ }^{48}$ M. Holden, K. Rodriguez, and J. Va, "An experimental study of transpiration cooling on the distribution of heat transfer and skin friction to a sharp slender cone at mach 11 and 13," in 28th Aerospace Sciences Meeting (1990) p. 308.

${ }^{49}$ M. Holden, R. Neumann, G. Burke, and K. Rodriguez, "An experimental study of the effects of injectant properties on the aerothermal characteristics of transpiration-cooled cones in hypersonic flow," in 21st Fluid Dynamics, Plasma Dynamics and Lasers Conference (1990) p. 1487.

${ }^{50} \mathrm{M}$. Holden, T. Wadhams, and E. Mundy, "A review of experimental studies of surface roughness and blowing on the heat transfer and skin friction to nosetips and slender cones in high mach numbers flows," in 40th Thermophysics Conference (2008) p. 3907.

${ }^{51}$ M. Holden, E. Mundy, and M. MacLean, "Heat transfer measurements to examine surface roughness and blowing effects in hypersonic flows," in 49th AIAA Aerospace Sciences Meeting including the New Horizons Forum and Aerospace Exposition (2011) p. 760.

${ }^{52}$ O. Zeman, "Dilatation dissipation: the concept and application in modeling compressible mixing layers," Physics of Fluids A: Fluid Dynamics 2, 178-188 (1990).

${ }^{53}$ S. Sarkar, G. Erlebacher, M. Y. Hussaini, and H. O. Kreiss, "The analysis and modelling of dilatational terms in compressible turbulence," Journal of Fluid Mechanics 227, 473-493 (1991).

${ }^{54}$ D. C. Wilcox, "Dilatation-dissipation corrections for advanced turbulence models," AIAA journal 30, 2639-2646 (1992).

${ }^{55} \mathrm{E}$. R. Van Driest, "On turbulent flow near a wall," Journal of the aeronautical sciences 23, 1007-1011 (1956).

${ }^{56}$ W. M. Kays, "Heat transfer to the transpired turbulent boundary layer," International Journal of Heat and Mass Transfer 15, 1023-1044 (1972).

${ }^{57}$ B. Aupoix, "Couches limites bidimensionnelles compressibles. descriptif et mode d'emploi du code clicet - version 2010," Report No. RT 1/117015 DMAE (ONERA, 2010).

${ }^{58} \mathrm{~F}$. R. Menter, "Two-equation eddy-viscosity turbulence models for engineering applications," AIAA Journal 32 (1994). 